\title{
Counting permutations with no long monotone subsequence via generating trees and the kernel method
}

\author{
Mireille Bousquet-Mélou
}

Received: 1 June 2010 / Accepted: 4 October 2010 / Published online: 22 October 2010

(C) Springer Science+Business Media, LLC 2010

\begin{abstract}
We recover Gessel's determinantal formula for the generating function of permutations with no ascending subsequence of length $m+1$. The starting point of our proof is the recursive construction of these permutations by insertion of the largest entry. This construction is of course extremely simple. The cost of this simplicity is that we need to take into account in the enumeration $m-1$ additional parameters-namely, the positions of the leftmost increasing subsequences of length $i$, for $i=2, \ldots, m$. This yields for the generating function a functional equation with $m-1$ "catalytic" variables, and the heart of the paper is the solution of this equation.

We perform a similar task for involutions with no descending subsequence of length $m+1$, constructed recursively by adding a cycle containing the largest entry. We refine this result by keeping track of the number of fixed points.

In passing, we prove that the ordinary generating functions of these families of permutations can be expressed as constant terms of rational series.
\end{abstract}

Keywords Permutations - Ascending subsequences · Generating functions · Generating trees

\section{Introduction}

Let $\tau=\tau(1) \cdots \tau(n)$ be a permutation in the symmetric group $\mathfrak{S}_{n}$. We denote by $|\tau|:=n$ the length of $\tau$. An ascending (resp. descending) subsequence of $\tau$ of length $k$ is a $k$-tuple $\left(\tau\left(i_{1}\right), \ldots, \tau\left(i_{k}\right)\right)$ such that $i_{1}<\cdots<i_{k}$ and $\tau\left(i_{1}\right)<\cdots<\tau\left(i_{k}\right)$ (resp. $\left.\tau\left(i_{1}\right)>\cdots>\tau\left(i_{k}\right)\right)$. For $m \geq 1$, the set of permutations in which all ascending subsequences have length at most $m$ is denoted by $\mathfrak{S}^{(m)}$. In pattern-avoidance terms, the

M. Bousquet-Mélou ( $\varangle)$

CNRS, LaBRI, Université Bordeaux 1, 351 cours de la Libération, 33405 Talence, France

e-mail: mireille.bousquet@labri.fr 
permutations of $\mathfrak{S}^{(m)}$ are those that avoid the increasing pattern $12 \cdots m(m+1)$, and an ascending subsequence of length $k$ is an occurrence of the pattern $12 \cdots k$. The set of $12 \cdots m(m+1)$-avoiding permutations of length $n$ is denoted $\mathfrak{S}_{n}^{(m)}$. Note that several families of pattern avoiding permutations are equinumerous with $\mathfrak{S}_{n}^{(m)}$ (see $[2,26])$.

In 1990, Gessel proved a beautiful determinantal formula for what could be called the Bessel generating function of permutations of $\mathfrak{S}^{(m)}$. This formula was the starting point of Baik, Deift and Johansson's study of the distribution of the longest ascending subsequence in a random permutation [4].

Theorem 1 ([16]) The Bessel generating function of permutations avoiding $12 \cdots m(m+1)$ is

$$
\sum_{\tau \in \mathfrak{S}^{(m)}} \frac{t^{2|\tau|}}{|\tau| !^{2}}=\operatorname{det}\left(I_{i-j}\right)_{1 \leq i, j \leq m},
$$

where

$$
I_{i}=\sum_{n \geq \max (0,-i)} \frac{t^{2 n+i}}{n !(n+i) !} .
$$

Note that $I_{i}=I_{-i}$, and that we can more loosely write

$$
I_{i}=\sum_{n \geq 0} \frac{t^{2 n+i}}{n !(n+i) !}=\sum_{n \geq 0} \frac{t^{2 n-i}}{n !(n-i) !},
$$

provided we interpret factorials as Gamma functions (in particular, $1 / i !=1 / \Gamma(i+$ 1) $=0$ if $i<0)$.

Gessel's original proof was algebraic in nature [16]. He first established a determinantal identity dealing with Schur functions (and hence with semi-standard Young tableaux, whereas the above theorem deals, via Schensted's correspondence, with standard tableaux). He then applied to this identity an operator $\theta$ that extracts certain coefficients, and this led to Theorem 1. A few years later, Krattenthaler found a bijective proof of Gessel's Schur function identity [23], which specializes into a bijective proof of Theorem 1. Then, Gessel, Weinstein and Wilf gave two bijective proofs of this theorem, involving sign-reversing involutions [18]. Two other proofs, involving Young tableaux, were recently published by Novak [30] and Xin [42].

For small values of $m$, more proofs of Theorem 1 have been given. In particular, there exists a wealth of ways of proving that the number of 123-avoiding permutations of $\mathfrak{S}_{n}$ is the $n^{\text {th }}$ Catalan number $\left(\begin{array}{c}2 n \\ n\end{array}\right) /(n+1)$, and numerous refinements of this result $[7,8,14,22,25,34-36,40]$. The laziest proof (combinatorially speaking) is based on the following observation: a permutation $\pi$ of $\mathfrak{S}_{n+1}^{(2)}$ is obtained by inserting $n+1$ in a permutation $\tau$ of $\mathfrak{S}_{n}^{(2)}$. To avoid the creation of an ascending subsequence of length 3 , the insertion must not take place to the right of the leftmost ascent of $\tau$. Hence, in order to exploit this simple recursive description of permutations of $\mathfrak{S}^{(2)}$, 
one must keep track of the position of the first ascent. Let us denote

$$
a(\tau)= \begin{cases}n+1, & \text { if } \tau \text { avoids } 12 \\ \min \{i: \tau(i-1)<\tau(i)\}, & \text { otherwise }\end{cases}
$$

and define the bivariate generating function

$$
F(u ; t):=\sum_{\tau \in \mathfrak{S}^{(2)}} u^{a(\tau)-1} t^{|\tau|} .
$$

It is not hard to see (and this will be explained in details in Sect. 2) that the recursive description of permutations of $\mathfrak{S}^{(2)}$ translates into the following equation:

$$
\left(1-t \frac{u^{2}}{u-1}\right) F(u ; t)=1-t \frac{u}{u-1} F(1 ; t) .
$$

The variable $u$ is said to be catalytic for this equation. This means that one cannot simply set $u=1$ to solve for $F(1 ; t)$ first. However, this equation can be solved using the so-called kernel method (see e.g., [5, 11, 33]): one specializes $u$ to the unique power series $U$ that cancels the kernel of the equation (that is, the coefficient of $F(u ; t))$ :

$$
U:=\frac{1-\sqrt{1-4 t}}{2 t} .
$$

This choice cancels the left-hand side of the equation, and thus its right-hand side, yielding the (ordinary) length generating function of 123-avoiding permutations:

$$
F(1 ; t)=\frac{U-1}{t U}=U=\frac{1-\sqrt{1-4 t}}{2 t}=\sum_{n \geq 0} \frac{t^{n}}{n+1}\left(\begin{array}{c}
2 n \\
n
\end{array}\right) .
$$

It is natural to ask whether this approach can be generalized to a generic value of $m$ : after all, a permutation $\pi$ of $\mathfrak{S}_{n+1}^{(m)}$ is still obtained by inserting $n+1$ in a permutation $\tau$ of $\mathfrak{S}_{n}^{(m)}$. However, to avoid creating an ascending subsequence of length $m+1$, the insertion must not take place to the right of the leftmost ascending subsequence of length $m$ of $\tau$. In order to keep track recursively of the position of this subsequence, one must also keep track of the position of the leftmost ascending subsequence of length $m-1$. And so on! Hence this recursive construction (often called the generating tree construction $[40,41])$ translates into a functional equation involving $m-1$ catalytic variables $u_{2}, \ldots, u_{m}$. The whole point is to solve this equation, and this is what we do in this paper. Our method combines three ingredients: an appropriate change of variables, followed by what is essentially the reflection principle [17], but performed at the level of power series, and finally a coefficient extraction. To warm up, we illustrate these ingredients in Sect. 3 by two simple examples: we first give another solution of (2) obtained when $m=2$, and then a generating function proof of MacMahon's formula for the number of standard tableaux of a given shape.

What is the interest of this exercise? Firstly, we believe it answers a natural question: we have in one hand a simple recursive construction of certain permutations, 
in the other hand a nice expression for their generating function, and it would be frustrating not to be able to derive the expression from the construction. Secondly, the combinatorial literature abounds in objects that can be described recursively by keeping track of an arbitrary (but bounded) number of additional (or: catalytic) parameters: permutations of course, but also lattice paths, tableaux, matchings, plane partitions, set partitions.... Some, but not all, can be solved by the reflection principle, and we hope that this first solution of an equation with $m$ catalytic variables will be followed by others.

In fact, we provide in this paper another application of our approach, still in the field of permutations: We recover a determinantal formula for the enumeration of involutions with no long descending subsequence [16]. Let $\mathfrak{I}^{(m)}$ (resp. $\mathfrak{I}_{n}^{(m)}$ ) denote the set of involutions (resp. involutions of length $n$ ) avoiding the decreasing pattern $(m+1) m \cdots 21$. Again, several families of pattern avoiding involutions are equinumerous with $\mathfrak{S}_{n}^{(m)}$ (see [12, 13, 21, 26]).

Theorem 2 The exponential generating function of involutions avoiding $(m+$ 1) $m \cdot 21$ is

$$
\sum_{\tau \in \mathfrak{I}^{(m)}} \frac{t^{|\tau|}}{|\tau| !}= \begin{cases}e^{t} \operatorname{det}\left(I_{i-j}-I_{i+j}\right)_{1 \leq i, j \leq \ell}, & \text { if } m=2 \ell+1 \\ \operatorname{det}\left(I_{i-j}+I_{i+j-1}\right)_{1 \leq i, j \leq \ell}, & \text { if } m=2 \ell\end{cases}
$$

where $I_{i}$ is defined by (1).

This result is obtained by applying Gessel's $\theta$ operator to a Schur function identity due to Bender and Knuth [6]. The latter identity has been refined by taking into account the number of columns of odd size in the tableaux (see Goulden [19]; Krattenthaler then gave a bijective proof of this refinement [23]). Using the operator $\theta$, and the properties of Schensted's correspondence [38, Exercise 7.28], this translates into a refinement of Theorem 2 that takes into account the number $f(\tau)$ of fixed points in $\tau$. We shall also recover this result.

Theorem 3 If $m=2 \ell+1$, the exponential generating function of involutions avoiding $(m+1) m \cdots 21$ and having $p$ fixed points is

$$
\sum_{\tau \in \mathfrak{I}^{(m)}, f(\tau)=p} \frac{t^{|\tau|}}{|\tau| !}=\frac{t^{p}}{p !} \operatorname{det}\left(I_{i-j}-I_{i+j}\right)_{1 \leq i, j \leq \ell} .
$$

If $m=2 \ell$, this generating function is

$$
\sum_{\substack{\tau \in \mathcal{I}^{(m)} \\
f(\tau)=p}} \frac{t^{|\tau|}}{|\tau| !}=\operatorname{det}\left(\begin{array}{c}
\left(I_{p+\ell-j}-I_{p+\ell+j}\right)_{1 \leq j \leq \ell} \\
\left(I_{i+j-1}-I_{i-j-1}\right)_{2 \leq i \leq \ell, 1 \leq j \leq \ell}
\end{array}\right),
$$

where we have described separately the first row of the determinant and the next $\ell-1$ rows $(i=2, \ldots, \ell)$. 
The first result of Theorem 3 can be restated as follows: if $m=2 \ell+1$, the generating function of involutions avoiding $(m+1) m \cdots 21$, counted by the length and number of fixed points is

$$
\sum_{\tau \in \mathfrak{I}^{(m)}} \frac{t^{|\tau|}}{|\tau| !} s^{f(\tau)}=e^{s t} \operatorname{det}\left(I_{i-j}-I_{i+j}\right)_{1 \leq i, j \leq \ell} .
$$

It thus appears as a very simple extension of the first part of Theorem 2, and indeed, the connection between these two formulas is easy to justify combinatorially (the fixed points play no role when one forbids a decreasing pattern of even length).

Let us now outline the structure of the paper. In Sect. 2, we describe how the "catalytic" parameters change in the recursive construction of permutations of $\mathfrak{S}^{(m)}$ and $\mathfrak{I}^{(m)}$. We do not give the proofs, as this was done by Guibert and Jaggard \& Marincel, respectively [20,21]. We then convert these descriptions into the functional equations that are at the heart of this paper (Propositions 5 and 7). In Sect. 3, we illustrate our approach by two simple examples, namely the enumeration of permutations of $\mathfrak{S}^{(2)}$ and of standard Young tableaux. Next we return to permutations: we first address in Sect. 4 the solution of the equation obtained for involutions of $\mathfrak{I}^{(m)}$, and finally, we solve in Sect. 5 the equation obtained for permutations of $\mathfrak{S}^{(m)}$. The reason why we address involutions first is that the solution is really elementary in this case. One step of the solution turns out to be more difficult in the case of permutations, although the basic ingredients are the same.

Let us finish with some standard definitions and notation. Let $A$ be a commutative ring and $x$ an indeterminate. We denote by $A[x]$ (resp. $A[[x]]$ ) the ring of polynomials (resp. formal power series) in $x$ with coefficients in $A$. If $A$ is a field, then $A(x)$ denotes the field of rational functions in $x$ (with coefficients in $A$ ). This notation is generalized to polynomials, fractions and series in several indeterminates. We denote $\bar{x}=1 / x$, so that $A[x, \bar{x}]$ is the ring of Laurent polynomials in $x$ with coefficients in $A$. A Laurent series is a series of the form $\sum_{n \geq n_{0}} a(n) x^{n}$, for some $n_{0} \in \mathbb{Z}$. The coefficient of $x^{n}$ in $F(x)$ is denoted $\left[x^{n}\right] F(x)$.

Most of the series that we use in this paper are power series in $t$ with coefficients in $A[x, \bar{x}]$, that is, series of the form

$$
F(x ; t)=\sum_{n \geq 0, i \in \mathbb{Z}} f(i ; n) x^{i} t^{n}
$$

where for all $n$, almost all coefficients $f(i ; n)$ are zero. The positive part of $F(x ; t)$ in $x$ is the following series, which has coefficients in $x A[x]$ :

$$
\left[x^{>}\right] F(x ; t):=\sum_{n \geq 0, i>0} f(i ; n) x^{i} t^{n} .
$$

We define similarly the negative, non-negative and non-positive parts of $F(x ; t)$ in $x$, which we denote respectively by $\left[x^{<}\right] F(x ; t),\left[x^{\geq}\right] F(x ; t)$ and $[x \leq] F(x ; t)$. 


\section{Generating trees and functional equations}

\subsection{Permutations avoiding $12 \cdots m(m+1)$}

Take a permutation $\pi$ of $\mathfrak{S}_{n+1}^{(m)}$, written as the word $\pi(1) \cdots \pi(n+1)$. Erase from this word the value $n+1$ : this gives a permutation $\tau$ of $\mathfrak{S}_{n}^{(m)}$. This property allows us to display the permutations of $\mathfrak{S}^{(m)}$ as the nodes of a generating tree. At the root of this tree sits the unique permutation of length 0 , and the children of a node indexed by $\tau \in \mathfrak{S}_{n}^{(m)}$ are the permutations of $\mathfrak{S}_{n+1}^{(m)}$ obtained by inserting the value $n+1$ in $\tau$. In how many ways is this insertion possible? If $\tau$ avoids $12 \cdots m$, then all insertion positions are admissible, that is, give a permutation of $\mathfrak{S}_{n+1}^{(m)}$. There are $n+1$ such positions. Otherwise, only the $a$ leftmost insertion positions are admissible, where $a$ is the position of the leftmost occurrence of $12 \cdots m$ in $\tau$. More precisely:

$$
a=\min \left\{i_{m}: \exists i_{1}<i_{2}<\cdots<i_{m} \text { s.t. } \tau\left(i_{1}\right)<\cdots<\tau\left(i_{m}\right)\right\} .
$$

As we wish to describe recursively the shape of the generating tree, we now need to find the position of the leftmost occurrence of $12 \cdots m$ in the children of $\tau$. But it is easily seen that this depends on the position of the leftmost occurrence of $12 \cdots(m-$ 1 ) in $\tau$. And so on! We are thus led to define the following $m$ parameters: for $1 \leq j \leq$ $m$, and $\tau \in \mathfrak{S}_{n}^{(m)}$, let

$a_{j}(\tau)= \begin{cases}n+1, & \text { if } \tau \text { avoids } 12 \cdots j \\ \min \left\{i_{j}: \exists i_{1}<i_{2}<\cdots<i_{j} \text { s.t. } \tau\left(i_{1}\right)<\cdots<\tau\left(i_{j}\right)\right\}, & \text { otherwise. }\end{cases}$

Note that $a_{1}(\tau)=1$, and that $a_{1}(\tau) \leq \cdots \leq a_{m}(\tau)$. We call the sequence $L(\tau):=$ $\left(a_{2}(\tau), \ldots, a_{m}(\tau)\right)$ the label of $\tau$. The empty permutation has label $(1, \ldots, 1)$.

We can now describe the labels of the children of $\tau$ in terms of $L(\tau)$ (Guibert [20, Prop. 4.47]).

Proposition 4 Let $\tau \in \mathfrak{S}_{n}^{(m)}$ with $L(\tau)=\left(a_{2}, \ldots, a_{m}\right)$. Denote $a_{1}=1$. The labels of the $a_{m}$ permutations of $\mathfrak{S}_{n+1}^{(m)}$ obtained by inserting $n+1$ in $\tau$ are

$$
\left\{\begin{array}{l}
\left(a_{2}+1, a_{3}+1, \ldots, a_{m}+1\right) \\
\left(a_{2}, \ldots, a_{j-1}, \alpha, a_{j+1}+1, \ldots, a_{m}+1\right)
\end{array} \text { for } 2 \leq j \leq m \text { and } a_{j-1}+1 \leq \alpha \leq a_{j} .\right.
$$

The first label corresponds to an insertion in position 1, while the label involving $\alpha$ corresponds to an insertion in position $\alpha$. We refer the reader to Fig. 1 for an example.

Let us now translate the recursive construction of permutations of $\mathfrak{S}^{(m)}$ in terms of generating functions. Let $\tilde{F}\left(u_{2}, \ldots, u_{m} ; t\right)$ be the (ordinary) generating function of permutations of $\mathfrak{S}^{(m)}$, counted by the statistics $a_{2}, \ldots, a_{m}$ and by the length:

$$
\begin{aligned}
\tilde{F}\left(u_{2}, \ldots, u_{m} ; t\right) & =\sum_{\tau \in \mathfrak{S}^{(m)}} u_{2}^{a_{2}(\tau)} \cdots u_{m}^{a_{m}(\tau)} t^{|\tau|} \\
& =\sum_{a_{2}, \ldots, a_{m}} \tilde{F}_{a_{2}, \ldots, a_{m}}(t) u_{2}^{a_{2}} \cdots u_{m}^{a_{m}},
\end{aligned}
$$


Fig. 1 The permutation $\tau=859613472 \in \mathfrak{S}_{9}^{(3)}$. One has $a_{1}(\tau)=1, a_{2}(\tau)=3$, $a_{3}(\tau)=7$. There are seven admissible ways to insert the value 10 . Inserting 10 to the right of $\tau$ (7) would create an occurrence of 1234

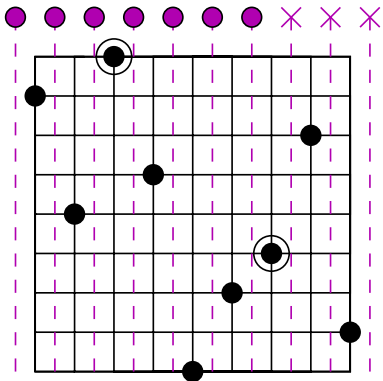

where $\tilde{F}_{a_{2}, \ldots, a_{m}}(t)$ is the length generating function of permutations of $\mathfrak{S}^{(m)}$ having label $\left(a_{2}, \ldots, a_{m}\right)$. We still denote $a_{1}=1$. The above proposition gives

$$
\begin{aligned}
& \tilde{F}\left(u_{2}, \ldots, u_{m} ; t\right) \\
& \quad=u_{2} \cdots u_{m}+t u_{2} \cdots u_{m} \tilde{F}\left(u_{2}, \ldots, u_{m} ; t\right) \\
& \quad+t \sum_{a_{2}, \ldots, a_{m}} \tilde{F}_{a_{2}, \ldots, a_{m}}(t) \sum_{j=2}^{m} \sum_{\alpha=a_{j-1}+1}^{a_{j}} u_{2}^{a_{2}} \cdots u_{j-1}^{a_{j-1}} u_{j}^{\alpha} u_{j+1}^{a_{j+1}+1} \cdots u_{m}^{a_{m}+1} .
\end{aligned}
$$

Using

$$
\sum_{\alpha=a_{j-1}+1}^{a_{j}} u_{j}^{\alpha}=\frac{u_{j}^{a_{j}+1}-u_{j}^{a_{j-1}+1}}{u_{j}-1},
$$

we obtain (given that $a_{1}=1$ )

$$
\begin{aligned}
\tilde{F}(u ; t)= & u_{2, m}+t u_{2, m} \tilde{F}(u ; t)+t u_{2, m} \frac{\tilde{F}(u ; t)-u_{2} \tilde{F}\left(1, u_{3}, \ldots, u_{m} ; t\right)}{u_{2}-1} \\
& +t \sum_{j=3}^{m} u_{j, m} \frac{\tilde{F}(u ; t)-\tilde{F}\left(u_{2}, \ldots, u_{j-2}, u_{j-1} u_{j}, 1, u_{j+1}, \ldots, u_{m} ; t\right)}{u_{j}-1},
\end{aligned}
$$

where $\tilde{F}(u ; t) \equiv \tilde{F}\left(u_{2}, \ldots, u_{m} ; t\right)$ and $u_{j, k}=u_{j} u_{j+1} \cdots u_{k}$.

To finish, let us perform an elementary transformation on the series $\tilde{F}(u ; t)$. Define

$$
F(v ; t)=F\left(v_{1}, \ldots, v_{m} ; t\right)=\sum_{\tau \in \mathfrak{S}^{(m)}} v_{1}^{a_{2}-1} v_{2}^{a_{3}-a_{2}} \cdots v_{m}^{|\tau|+1-a_{m}} t^{|\tau|}
$$

where $\left(a_{2}, \ldots, a_{m}\right)=L(\tau)$. We have eliminated the dependence $a_{2} \leq \cdots \leq a_{m}$ between the exponents of $u_{2}, \ldots, u_{m}$ in $\tilde{F}(u ; t)$. As will be seen below, another effect of this change of series is that the cases $j=2$ and $j=3, \ldots, m$ now play the same role. We also note that the variable $t$ is now redundant in $F(v ; t)$, but it is our main variable, and we find it convenient to keep it. The series $\tilde{F}$ and $F$ are related by

$$
F\left(v_{1}, \ldots, v_{m} ; t\right)=\frac{v_{m}}{v_{1}} \tilde{F}\left(\frac{v_{1}}{v_{2}}, \ldots, \frac{v_{m-1}}{v_{m}} ; v_{m} t\right)
$$


and conversely

$$
\tilde{F}\left(u_{2}, \ldots, u_{m} ; v_{m} t\right)=u_{2, m} F\left(u_{2, m} v_{m}, u_{3, m} v_{m}, \ldots, u_{m} v_{m}, v_{m} ; t\right),
$$

where as above $u_{j, k}=u_{j} u_{j+1} \cdots u_{k}$. The functional equation (5) satisfied by $\tilde{F}(u ; t)$ translates into an equation of a slightly simpler form satisfied by $F(v ; t)$.

Proposition 5 The generating function $F(v ; t) \equiv F\left(v_{1}, \ldots, v_{m} ; t\right)$ of permutations of $\mathfrak{S}^{(m)}$, defined by (6), satisfies

$$
\begin{aligned}
F(v ; t)=1 & +t v_{1} F(v ; t) \\
& +t \sum_{j=2}^{m} v_{j-1} v_{j} \frac{F(v ; t)-F\left(v_{1}, \ldots, v_{j-2}, v_{j}, v_{j}, v_{j+1}, \ldots, v_{m} ; t\right)}{v_{j-1}-v_{j}} .
\end{aligned}
$$

The series $F(1, \ldots, 1 ; t)$ counts permutations of $\mathfrak{S}^{(m)}$ by their length.

In Sect. 5, we derive from this equation the Bessel generating function of permutations of $\mathfrak{S}^{(m)}$, as given by Theorem 1 .

\subsection{Involutions avoiding $(m+1) m \cdots 21$}

It follows from the properties of Schensted's correspondence [37] that the number of involutions of length $n$ avoiding $12 \cdots m(m+1)$ equals the number of involutions of length $n$ avoiding $(m+1) m \cdots 21$. However, this correspondence is not a simple symmetry, and the generating trees that describe $12 \cdots m(m+1)$-avoiding involutions and $(m+1) m \cdots 21$-avoiding involutions are not isomorphic. Both trees are defined by the same principle: the root is the empty permutation and the parent of an involution $\pi$ is obtained by deleting the cycle containing the largest entry, and normalizing the resulting sequence. For instance, if $\pi=426153$, the deletion of the 2-cycle $(3,6)$ first gives 4215, and, after normalization, 3214.

The tree that generates $12 \cdots m(m+1)$-avoiding involutions is similar to the tree generating $12 \cdots m(m+1)$-avoiding permutations. Its description involves $m$ catalytic parameters (Guibert [20, Prop. 4.52]). The tree that generates $(m+1) m \cdots 21$ avoiding involutions requires $\lfloor m / 2\rfloor$ catalytic parameters only (Jaggard \& Marincel [21]). The source of this compactness is easy to understand: an involution $\tau$ contains the pattern $k \cdots 21$ if and only if it contains a symmetric occurrence of this pattern (by this, we mean that the corresponding set of points in the diagram of $\tau$ is symmetric with respect to the first diagonal, see Fig. 2). Equivalently, this means that a decreasing subsequence of length $\lceil k / 2\rceil$ occurs in the points of the diagram lying on or above the first diagonal. Thus we only need to keep track of descending subsequences of length at most $m / 2$ (in the top part of the diagram), and we can expect to have about $m / 2$ catalytic parameters.

Let us now describe in details the tree generating $(m+1) m \cdots 21$-avoiding involutions. The example of Fig. 2 illustrates the argument. Let $\tau$ be an involution of $\mathfrak{I}_{n}^{(m)}$. Inserting $n+1$ as a fixed point in $\tau$ always gives an involution of $\mathfrak{I}^{(m)}$. For 
Fig. 2 The involution $\tau=321127958611104 \in \mathfrak{I}_{12}^{(5)}$.

One has $a_{1}(\tau)=3, a_{2}(\tau)=9$.

There are nine admissible ways to insert a 2-cycle

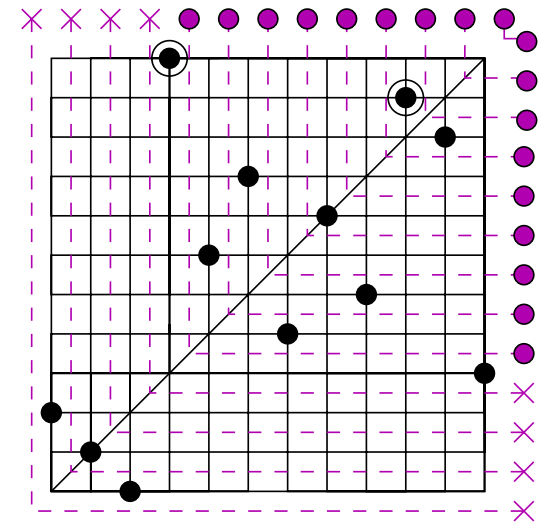

$1 \leq i \leq n+1$, let us now consider the permutation $\pi$ obtained by adding 1 to all values larger than or equal to $i$, and inserting the 2-cycle $(i, n+2)$. How many of these insertions are admissible, that is, give an involution of $\mathfrak{I}^{(m)}$ ? If $\tau$ avoids $(m-1) \cdots 21$, then all insertions are admissible, including the most "risky" one, corresponding to $i=1$. Otherwise, the only admissible values of $i$ are $n+1, n, \ldots, n-a+2$, where $n-a+1$ is the position of the rightmost symmetric occurrence of $(m-1) \cdots 21$. In other words, if we denote $m=2 \ell+\epsilon$ with $\epsilon \in\{0,1\}$,

$$
n-a+1=\max \left\{i_{1}: \exists i_{1}<i_{2}<\cdots<i_{\ell} \text { s.t. } \tau\left(i_{1}\right)>\cdots>\tau\left(i_{\ell}\right) \geq i_{\ell}+\epsilon\right\} .
$$

Again, in order to keep track of this parameter recursively, we are led to define, for $1 \leq j \leq \ell$, the following $\ell$ catalytic parameters:

$$
a_{j}(\tau)=\left\{\begin{array}{l}
n+1, \quad \text { if } \tau \text { avoids }(2 j-1+\epsilon) \cdots 21 \\
n+1-\max \left\{i_{1}: \exists i_{1}<i_{2}<\cdots<i_{j} \text { s.t. } \tau\left(i_{1}\right)>\cdots>\tau\left(i_{j}\right) \geq i_{j}+\epsilon\right\}, \\
\quad \text { otherwise. }
\end{array}\right.
$$

In particular, $a_{\ell}(\tau)$ is the parameter that was denoted $a$ above, and it is also the number of admissible insertions of a 2-cycle in $\tau$. We call the sequence $L(\tau):=$ $\left(a_{1}(\tau), \ldots, a_{\ell}(\tau)\right)$ the label of $\tau$. Note that $a_{1}(\tau) \leq \cdots \leq a_{\ell}(\tau)$. The empty permutation has label $(1, \ldots, 1)$.

We can now describe the labels of the children of $\tau$ in terms of $L(\tau)$.

Proposition 6 (Jaggard \& Marincel [21]) Let $\tau$ be an involution in $\mathfrak{I}^{(m)}$ with $L(\tau)=$ $\left(a_{1}, \ldots, a_{\ell}\right)$. Denote $a_{0}=0$. The labels of the $a_{\ell}$ involutions of $\mathfrak{I}^{(m)}$ obtained by inserting a cycle in $\tau$ are

$$
\begin{cases}\left(a_{1}+1, a_{2}+1, \ldots, a_{\ell}+1\right), & \text { if } m \text { is odd } \\ \left(1, a_{2}+1, \ldots, a_{\ell}+1\right), & \text { if } m \text { is even } \\ \left(a_{1}+1, \ldots, a_{j-1}+1, \alpha, a_{j+1}+2, \ldots, a_{\ell}+2\right) & \\ \text { for } 1 \leq j \leq \ell \text { and } a_{j-1}+2 \leq \alpha \leq a_{j}+1 . & \end{cases}
$$

The first two labels correspond to the insertion of a fixed point, the other ones to the insertion of a 2-cycle. 
We refer again the reader to Fig. 2 for an example.

Let us now translate the recursive construction of involutions of $\mathfrak{I}^{(m)}$ in terms of generating functions. Let $\tilde{G}\left(u_{1}, \ldots, u_{\ell} ; t\right)$ be the (ordinary) generating function of involutions of $\mathfrak{I}^{(m)}$, counted by the statistics $a_{1}, \ldots, a_{\ell}$ and by the length:

$$
\begin{aligned}
\tilde{G}\left(u_{1}, \ldots, u_{\ell} ; t\right) & =\sum_{\tau \in \mathfrak{I}^{(m)}} u_{1}^{a_{1}(\tau)} \cdots u_{\ell}^{a_{\ell}(\tau)} t^{|\tau|} \\
& =\sum_{a_{1}, \ldots, a_{\ell}} \tilde{G}_{a_{1}, \ldots, a_{\ell}}(t) u_{1}^{a_{1}} \cdots u_{\ell}^{a_{\ell}},
\end{aligned}
$$

where $\tilde{G}_{a_{1}, \ldots, a_{\ell}}(t)$ is the length generating function of permutations of $\mathfrak{I}^{(m)}$ having label $\left(a_{1}, \ldots, a_{\ell}\right)$. We still denote $a_{0}=0$. The above proposition gives

$$
\begin{aligned}
& \tilde{G}\left(u_{1}, \ldots, u_{\ell} ; t\right) \\
& =u_{1} \cdots u_{\ell}+t u_{1} \cdots u_{\ell} \tilde{G}\left(u_{1}, \ldots, u_{\ell} ; t\right) \chi_{m \equiv 1}+t u_{1} \cdots u_{\ell} \tilde{G}\left(1, u_{2}, \ldots, u_{\ell} ; t\right) \chi_{m \equiv 0} \\
& \quad+t^{2} \sum_{a_{1}, \ldots, a_{\ell}} \tilde{G}_{a_{1}, \ldots, a_{\ell}}(t) \sum_{j=1}^{\ell} \sum_{\alpha=a_{j-1}+2}^{a_{j}+1} u_{1}^{a_{1}+1} \cdots u_{j-1}^{a_{j-1}+1} u_{j}^{\alpha} u_{j+1}^{a_{j+1}+2} \cdots u_{\ell}^{a_{\ell}+2},
\end{aligned}
$$

where $\chi_{m \equiv i}$ equals 1 if $m$ equals $i$ modulo 2, and 0 otherwise. Using

$$
\sum_{\alpha=a_{j-1}+2}^{a_{j}+1} u_{j}^{\alpha}=\frac{u_{j}^{a_{j}+2}-u_{j}^{a_{j-1}+2}}{u_{j}-1}
$$

we finally obtain (given that $a_{0}=0$ )

$$
\begin{aligned}
\tilde{G}(u ; t)= & u_{1, \ell}+t u_{1, \ell} \tilde{G}(u ; t) \chi_{m \equiv 1}+t u_{1, \ell} \tilde{G}\left(1, u_{2}, \ldots, u_{\ell} ; t\right) \chi_{m \equiv 0} \\
& +t^{2} u_{1, \ell} \sum_{j=1}^{\ell} u_{j, \ell} \frac{\tilde{G}(u ; t)-\tilde{G}\left(u_{1}, \ldots, u_{j-2}, u_{j-1} u_{j}, 1, u_{j+1}, \ldots, u_{\ell} ; t\right)}{u_{j}-1},
\end{aligned}
$$

where $\tilde{G}(u ; t) \equiv \tilde{G}\left(u_{1}, \ldots, u_{\ell} ; t\right)$ and $u_{j, k}=u_{j} u_{j+1} \cdots u_{k}$.

To finish, let us perform an elementary transformation on the series $\tilde{G}(u ; t)$. Define

$$
G(v ; t)=G\left(v_{1}, \ldots, v_{\ell} ; t\right)=\sum_{\tau \in \mathfrak{I}^{(m)}} v_{1}^{a_{1}} v_{2}^{a_{2}-a_{1}} \cdots v_{\ell}^{a_{\ell}-a_{\ell-1}} t^{|\tau|},
$$

where $\left(a_{1}, \ldots, a_{\ell}\right)=\ell(\tau)$. We have eliminated the dependence $a_{1} \leq \cdots \leq a_{\ell}$ between the exponents of $u_{1}, \ldots, u_{\ell}$ in $\tilde{G}(u ; t)$. The series $\tilde{G}$ and $G$ are related by

$$
G\left(v_{1}, \ldots, v_{\ell} ; t\right)=\tilde{G}\left(\frac{v_{1}}{v_{2}}, \ldots, \frac{v_{\ell-1}}{v_{\ell}}, v_{\ell} ; t\right),
$$

and conversely

$$
\tilde{G}\left(u_{1}, \ldots, u_{\ell} ; t\right)=G\left(u_{1, \ell}, u_{2, \ell}, \ldots, u_{\ell} ; t\right),
$$


where as above $u_{j, k}=u_{j} u_{j+1} \cdots u_{k}$. The functional equation (7) satisfied by $\tilde{G}(u ; t)$ translates as follows.

Proposition 7 The generating function $G(v ; t) \equiv G\left(v_{1}, \ldots, v_{\ell} ; t\right)$ of involutions of $\mathfrak{I}^{(m)}$, defined by (8), satisfies

$$
\begin{aligned}
G(v ; t)= & v_{1}+t v_{1} G(v ; t) \chi_{m \equiv 1}+t v_{1} G\left(v_{2}, v_{2}, v_{3}, \ldots, v_{\ell} ; t\right) \chi_{m \equiv 0} \\
& +t^{2} v_{1} \sum_{j=1}^{\ell} v_{j} v_{j+1} \frac{G(v ; t)-G\left(v_{1}, \ldots, v_{j-1}, v_{j+1}, v_{j+1}, v_{j+2}, \ldots, v_{\ell} ; t\right)}{v_{j}-v_{j+1}}
\end{aligned}
$$

The series $G(1, \ldots, 1 ; t)$ counts involutions of $\mathfrak{I}^{(m)}$ by their length.

In Sect. 4, we derive from this equation the exponential generating function of involutions of $\mathfrak{I}^{(m)}$, as given by Theorem 2 . We then refine the result to take into account the number of fixed points.

\section{Two examples}

In this section, we illustrate the ingredients of our solution of the equations of Propositions 5 and 7 by taking two examples. The first one deals with the enumeration of 123 -avoiding permutations. The second one is a generating function proof of MacMahon's formula for the number of standard tableaux of a given shape, and should clarify what we meant in the introduction by "the reflection principle performed at the level of power series".

\subsection{Permutations avoiding 123}

In the introduction, we wrote the following equation for the bivariate generating function of 123-avoiding permutations, counted by the position of the first ascent and the length:

$$
\left(1-t \frac{u^{2}}{u-1}\right) F(u ; t)=1-t \frac{u}{u-1} F(1 ; t) .
$$

This is the case $m=2$ of Proposition 5, with $v_{1}=u$ and $v_{2}=1$.

As explained in Sect. 1, this equation can be solved by an appropriate choice of $u$ that cancels the kernel, and thus eliminates the unknown series $F(u ; t)$. This is the standard kernel method. We present here an alternative solution, sometimes called the algebraic kernel method $[9,10]$, where instead $F(1 ; t)$ is eliminated. This elimination is obtained by exploiting a certain symmetry of the kernel. This symmetry appears clearly if we set $u=1+x$. The equation then reads:

$$
(1-t(1+x)(1+\bar{x})) F(1+x ; t)=1-t(1+\bar{x}) F(1 ; t)
$$

with $\bar{x}=1 / x$. The kernel is now invariant under $x \mapsto \bar{x}$. Replace $x$ by $\bar{x}$ :

$$
(1-t(1+x)(1+\bar{x})) F(1+\bar{x} ; t)=1-t(1+x) F(1 ; t) .
$$


We now eliminate $F(1 ; t)$ by taking a linear combination of these two equations. This leaves

$$
(1-t(1+x)(1+\bar{x}))(F(1+x ; t)-\bar{x} F(1+\bar{x} ; t))=1-\bar{x}
$$

or

$$
F(1+x ; t)-\bar{x} F(1+\bar{x} ; t)=\frac{1-\bar{x}}{1-t(1+x)(1+\bar{x})}:=R(x ; t) .
$$

In this equation,

- $F(1+x ; t)$ is a series in $t$ with coefficients in $\mathbb{Q}[x]$,

$-\bar{x} F(1+\bar{x} ; t)$ is a series in $t$ with coefficients in $\bar{x} \mathbb{Q}[\bar{x}]$,

- the right-hand side $R(x ; t)$ is a series in $t$ with coefficients in $\mathbb{Q}[x, \bar{x}]$.

Consequently, $F(1+x ; t)$ is the non-negative part of $R(x ; t)$ in $x$. In particular, the length generating function of 123 -avoiding permutations is

$$
\begin{aligned}
F(1 ; t)=\left[x^{0}\right] R(x ; t) & =\sum_{n \geq 0}\left[x^{0}\right](1-\bar{x}) \bar{x}^{n}(1+x)^{2 n} t^{n} \\
& =\sum_{n \geq 0}\left(\left(\begin{array}{c}
2 n \\
n
\end{array}\right)-\left(\begin{array}{c}
2 n \\
n+1
\end{array}\right)\right) t^{n} \\
& =\sum_{n \geq 0} \frac{t^{n}}{n+1}\left(\begin{array}{c}
2 n \\
n
\end{array}\right) .
\end{aligned}
$$

This small example contains all ingredients of what will be our solution for a generic value of $m$ :

- a change of variables, which may not have a clear combinatorial meaning,

- a finite group $G$ acting on power series that leaves the kernel unchanged (here, the group has order 2 , and replaces $x$ by $1 / x$ ),

- a linear combination (9) of all the equations obtained by letting an element of $G$ act on the original functional equation; in this linear combination, called the orbit sum, the left-hand side is a multiple of the kernel, and the right-hand side does not contain any unknown series,

- finally, a coefficient extraction (10) that gives the generating function under interest.

Let us mention, however, that for a generic value of $m$, the change of variables used in Sect. 5 is not a direct extension of $v \mapsto 1+x$. But, on this small example, the latter choice is simpler.

\subsection{Standard Young tableaux}

Let $\lambda=\left(\lambda_{1}, \ldots, \lambda_{m}\right) \in \mathbb{N}^{m}$ be an integer partition. That is, $\lambda_{1} \geq \cdots \geq \lambda_{m} \geq 0$. The weight of $\lambda$ is $|\lambda|:=\lambda_{1}+\cdots+\lambda_{m}$. We identify $\lambda$ with its Ferrers shape, in which the $i^{\text {th }}$ row has $\lambda_{i}$ cells. A standard tableau of shape $\lambda$ is a filling of the cells of $\lambda$ with the integers $1,2, \ldots,|\lambda|$, that increases along rows and columns (Fig. 3). The 
Fig. 3 The Ferrers shape associated with the partition $\lambda=(4,3,3)$ and a standard tableau of shape $\lambda$

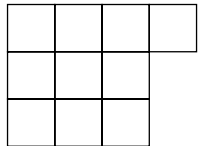

\begin{tabular}{|l|l|l|l|}
\hline 1 & 2 & 4 & 8 \\
\hline 3 & 5 & 9 & \multicolumn{1}{|c}{} \\
\cline { 1 - 2 } 6 & 7 & 10 &
\end{tabular}

height of the tableau is the number of non-empty rows, that is $\max \left(i: \lambda_{i}>0\right)$. Let $f^{\lambda}$ denote the number of standard Young tableaux of shape $\lambda$.

Our objective here is to recover the hook-length formula, or, rather, an equivalent form due to MacMahon [29, Sect. III, Chap. V].

Proposition 8 Let $\lambda=\left(\lambda_{1}, \ldots, \lambda_{m}\right)$ be a partition of weight $n$. The number of standard Young tableaux of shape $\lambda$ is

$$
f^{\lambda}=\frac{n !}{\prod_{i=1}^{m}\left(\lambda_{i}-i+m\right) !} \prod_{1 \leq i<j \leq m}\left(\lambda_{i}-\lambda_{j}-i+j\right) .
$$

Proof Let $F(u) \equiv F\left(u_{1}, \ldots, u_{m}\right)$ be the generating function of standard tableaux of height at most $m$ :

$$
F(u):=\sum_{\lambda_{1} \geq \cdots \geq \lambda_{m} \geq 0} f^{\lambda} \prod_{i=1}^{m} u_{i}^{\lambda_{i}} .
$$

For $j=2, \ldots, m$, we denote by $F_{j}\left(u_{1}, \ldots, u_{j-2}, u_{j-1} u_{j}, u_{j+1}, \ldots, u_{m}\right) \equiv F_{j}(u)$ the generating function of standard tableaux such that the parts $\lambda_{j-1}$ and $\lambda_{j}$ are equal. This series is obtained by extracting the corresponding terms from $F(u)$ (it is also called the $(j-1, j)$-diagonal of $F(u))$. In all terms of this series, $u_{j-1}$ and $u_{j}$ appear with the same exponent, which allows us to write this series in the above form.

Now a tableau of weight $n+1$ is obtained by adding a cell labeled $n+1$ to a tableau of weight $n$. This cell can be added to the $j^{\text {th }}$ row unless this row should have the same length as the $(j-1)^{\text {st }}$ row. This gives directly the following equation:

$$
F(u)=1+u_{1} F(u)+\sum_{j=2}^{m} u_{j}\left(F(u)-F_{j}(u)\right),
$$

that is,

$$
\left(1-\sum_{j=1}^{m} u_{j}\right) F(u)=1-\sum_{j=2}^{m} u_{j} F_{j}(u)
$$

Observe that the kernel $K(u):=1-\sum u_{j}$ is invariant under the action of the symmetric group $\mathfrak{S}_{m}$, seen as a group of transformations of polynomials in $u_{1}, \ldots, u_{m}$. This group is generated by $m-1$ elements of order 2 , denoted $\sigma_{1}, \ldots, \sigma_{m-1}$ :

$$
\sigma_{j}\left(P\left(u_{1}, \ldots, u_{m}\right)\right)=P\left(u_{1}, \ldots, u_{j-1}, u_{j+1}, u_{j}, u_{j+2}, \ldots, u_{m}\right) .
$$


Let us multiply the equation by $M(u):=u_{1}^{m-1} \cdots u_{m-1}^{1} u_{m}^{0}$. This gives:

$$
K(u) M(u) F(u)=M(u)-\sum_{j=2}^{m} u_{1}^{m-1} \cdots u_{j-1}^{m-(j-1)} u_{j}^{m-j+1} \cdots u_{m}^{0} F_{j}(u)
$$

Recall that $F_{j}(u)$ stands for $F_{j}\left(u_{1}, \ldots, u_{j-2}, u_{j-1} u_{j}, u_{j+1}, \ldots, u_{m}\right)$. Hence the $j^{\text {th }}$ term in the above sum is invariant under the action of the generator $\sigma_{j-1}$ (which exchanges $u_{j-1}$ and $u_{j}$ ). Consequently, forming the signed sum of (11) over the symmetric group $\mathfrak{S}_{m}$ gives the following orbit sum, which does not involve the series $F_{j}$ :

$$
\sum_{\sigma \in \mathfrak{S}_{m}} \varepsilon(\sigma) \sigma(K(u) M(u) F(u))=\sum_{\sigma \in \mathfrak{S}_{m}} \varepsilon(\sigma) \sigma(M(u)),
$$

or, given that $K(u)$ is $\mathfrak{S}_{m}$-invariant,

$$
\sum_{\sigma \in \mathfrak{S}_{m}} \varepsilon(\sigma) \sigma(M(u) F(u))=\frac{\sum_{\sigma \in \mathfrak{S}_{m}} \varepsilon(\sigma) \sigma(M(u))}{K(u)} .
$$

Of course, the sum on the right-hand side can be evaluated explicitly (the numerator is the Vandermonde determinant), but this will not be needed here.

We claim that the number $f^{\lambda}$ can be simply obtained by a coefficient extraction in the above identity. Consider the series $M(u) F(u)$. Each monomial $u_{1}^{a_{1}} \cdots u_{m}^{a_{m}}$ that occurs in it satisfies $a_{1}>\cdots>a_{m}$ (because $a_{i}=m-i+\lambda_{i}$, where $\lambda$ is a partition). Consequently, if $\sigma$ is not the identity, the exponents of any monomial $u_{1}^{a_{1}} \cdots u_{m}^{a_{m}}$ occurring in $\sigma(M(u) F(u))$ are totally ordered in a different way. Hence, when we extract the coefficient of $u_{1}^{m-1+\lambda_{1}} \cdots u_{m}^{0+\lambda_{m}}$ from (12), only the term corresponding to $\sigma=$ id contributes in the left-hand side, so that

$$
f^{\lambda}=\left[u_{1}^{m-1+\lambda_{1}} \cdots u_{m}^{0+\lambda_{m}}\right] \frac{\sum_{\sigma \in \mathfrak{S}_{m}} \varepsilon(\sigma) \sigma(M(u))}{K(u)} .
$$

Given that $M(u)=u_{1}^{m-1} \cdots u_{m-1}^{1} u_{m}^{0}$ and

$$
\frac{1}{K(u)}=\frac{1}{1-\sum_{j=1}^{m} u_{j}}=\sum_{a_{1}, \ldots, a_{m} \geq 0} \frac{\left(a_{1}+\cdots+a_{m}\right) !}{\prod_{i=1}^{m} a_{i} !} u_{1}^{a_{1}} \cdots u_{m}^{a_{m}},
$$

we obtain

$$
\begin{aligned}
f^{\lambda} & =\sum_{\sigma \in \mathfrak{S}_{m}} \varepsilon(\sigma) \frac{\left(\lambda_{1}+\cdots+\lambda_{m}\right) !}{\prod_{i=1}^{m}\left(\lambda_{i}-i+\sigma^{-1}(i)\right) !} \\
& =n ! \operatorname{det}\left(\frac{1}{\left(\lambda_{i}-i+j\right) !}\right)_{1 \leq i, j \leq m} \\
& =n ! \operatorname{det}\left(\frac{\left(\lambda_{i}-i+j+1\right) \cdots\left(\lambda_{i}-i+m\right)}{\left(\lambda_{i}-i+m\right) !}\right)_{1 \leq i, j \leq m}
\end{aligned}
$$




$$
=\frac{n !}{\prod_{i=1}^{m}\left(\lambda_{i}-i+m\right) !} \operatorname{det}\left(\left(\lambda_{i}-i+j+1\right) \cdots\left(\lambda_{i}-i+m\right)\right)_{1 \leq i, j \leq m} .
$$

The $(i, j)$-coefficient of the latter determinant is a polynomial in $\lambda_{i}-i$ of degree $m-j$ and leading coefficient 1 . Hence the determinant is simply the Vandermonde determinant $\operatorname{det}\left(\left(\lambda_{i}-i\right)^{m-j}\right)$, that is, $\prod_{i<j}\left(\lambda_{i}-\lambda_{j}-i+j\right)$. This completes the proof of the proposition.

We recognize in this proof three of the four ingredients that were used in the enumeration of 123-avoiding permutations: the finite group that leaves the kernel invariant (here, $\mathfrak{S}_{m}$ ), the orbit sum (12), and the final coefficient extraction. In this example, the symmetries of the kernel are obvious already with the original variables $u_{i}$, so that no change of variables is required.

This proof is the generating function counterpart of the classical proof that encodes tableaux of height at most $m$ by paths in $\mathbb{N}^{m}$ formed of unit positive steps, that start from $(0, \ldots, 0)$ and remain in the wedge $x_{1} \geq \cdots \geq x_{m} \geq 0$, and then uses the reflection principle. It is also very close to another proof due to Xin [42, Sect. 3.1].

\section{Involutions with no long descending subsequence}

We now address the solution of the functional equation of Proposition 7, which defines the generating function of involutions avoiding $(m+1) m \cdots 21$.

\subsection{Invariance properties of the kernel}

As discussed in the previous section, our objective is to exploit invariance properties of the kernel, that is, the coefficient of $G(v ; t)$. Let us first divide the equation of Proposition 7 by $v_{1}$. Then the kernel reads

$$
\frac{1}{v_{1}}-t \chi_{m \equiv 1}-t^{2} \sum_{j=1}^{\ell} \frac{v_{j} v_{j+1}}{v_{j}-v_{j+1}}
$$

The invariance properties of this rational function appear clearly after performing the following change of variables:

$$
v_{i}=\frac{1}{1-t\left(x_{i}+\cdots+x_{\ell}\right)} .
$$

Indeed, the kernel becomes

$$
K(x ; t)=1-t\left(x_{1}+\cdots+x_{\ell}\right)-t \chi_{m \equiv 1}-t\left(\bar{x}_{1}+\cdots+\bar{x}_{\ell}\right),
$$

where $\bar{x}_{i}=1 / x_{i}$, and is invariant under the action of the hyperoctahedral group $B_{\ell}$ (the group of signed permutations), seen as a group of transformations on Laurent 
polynomials in $x_{1}, \ldots, x_{\ell}$. This group is generated by $\ell$ elements of order 2 , denoted $\sigma_{1}, \ldots, \sigma_{\ell}$ :

$$
\sigma_{j}\left(P\left(x_{1}, \ldots, x_{\ell}\right)\right)= \begin{cases}P\left(\bar{x}_{1}, x_{2}, \ldots, x_{\ell}\right), & \text { if } j=1 ; \\ P\left(x_{1}, \ldots, x_{j-2}, x_{j}, x_{j-1}, x_{j+1}, \ldots, x_{\ell}\right), & \text { for } j \geq 2 .\end{cases}
$$

The equation of Proposition 7 now reads:

$$
\begin{aligned}
K(x ; t) \bar{G}(x ; t)= & 1+t \bar{G}\left(0, x_{2}, \ldots, x_{\ell}\right) \chi_{m \equiv 0} \\
& -t \sum_{j=1}^{\ell} \bar{x}_{j} \bar{G}\left(x_{1}, \ldots, x_{j-2}, x_{j-1}+x_{j}, 0, x_{j+1}, \ldots, x_{\ell}\right),
\end{aligned}
$$

where

$$
\begin{aligned}
\bar{G}(x ; t) & \equiv \bar{G}\left(x_{1}, \ldots, x_{\ell} ; t\right) \\
& =G\left(\frac{1}{1-t\left(x_{1}+\cdots+x_{\ell}\right)}, \frac{1}{1-t\left(x_{2}+\cdots+x_{\ell}\right)}, \ldots, \frac{1}{1-t x_{\ell}} ; t\right) .
\end{aligned}
$$

\subsection{Orbit sum}

We now handle separately the odd and even case.

- If $m$ is odd, the equation reads

$$
K(x ; t) \bar{G}(x ; t)=1-t \sum_{j=1}^{\ell} \bar{x}_{j} \bar{G}\left(x_{1}, \ldots, x_{j-2}, x_{j-1}+x_{j}, 0, x_{j+1}, \ldots, x_{\ell} ; t\right),
$$

where

$$
K(x ; t)=1-t\left(1+x_{1}+\cdots+x_{\ell}+\bar{x}_{1}+\cdots+\bar{x}_{\ell}\right) .
$$

Let us multiply the equation by

$$
M(x):=x_{1} x_{2}^{2} \cdots x_{\ell}^{\ell} .
$$

This gives

$$
\begin{aligned}
& K(x ; t) M(x) \bar{G}(x ; t) \\
& \quad=M(x)-t \sum_{j=1}^{\ell} x_{1} \cdots x_{j-1}^{j-1} x_{j}^{j-1} x_{j+1}^{j+1} \cdots x_{\ell}^{\ell} \\
& \quad \times \bar{G}\left(x_{1}, \ldots, x_{j-2}, x_{j-1}+x_{j}, 0, x_{j+1}, \ldots, x_{\ell} ; t\right) .
\end{aligned}
$$

The first term $(j=1)$ of the sum reads $x_{2}^{2} \cdots x_{\ell}^{\ell} \bar{G}\left(0, x_{2} \ldots, x_{\ell}\right)$ and is invariant under the action of the generator $\sigma_{1}$ of $B_{\ell}$ (which replaces $x_{1}$ by $\bar{x}_{1}$ ). For $j \geq 2$, the $j^{\text {th }}$ term of the sum is invariant under the action of the generator $\sigma_{j}$ (which exchanges 
$x_{j-1}$ and $x_{j}$ ). Consequently, forming the signed sum of (16) over the hyperoctahedral group $B_{\ell}$ gives the following orbit sum:

$$
\sum_{\sigma \in B_{\ell}} \varepsilon(\sigma) \sigma(K(x ; t) M(x) \bar{G}(x ; t))=\sum_{\sigma \in B_{\ell}} \varepsilon(\sigma) \sigma(M(x)),
$$

or, given that $K(x ; t)$ is $B_{\ell}$-invariant,

$$
\sum_{\sigma \in B_{\ell}} \varepsilon(\sigma) \sigma(M(x) \bar{G}(x ; t))=\frac{\sum_{\sigma \in B_{\ell}} \varepsilon(\sigma) \sigma(M(x))}{K(x ; t)}
$$

where $K(x ; t)$ is given by (14) and $M(x)$ by (15).

- If $m$ is even, the equation reads

$$
\begin{aligned}
K(x ; t) \bar{G}(x ; t)= & 1+t\left(1-\bar{x}_{1}\right) \bar{G}\left(0, x_{2}, \ldots, x_{\ell} ; t\right) \\
& -t \sum_{j=2}^{\ell} \bar{x}_{j} \bar{G}\left(x_{1}, \ldots, x_{j-2}, x_{j-1}+x_{j}, 0, x_{j+1}, \ldots, x_{\ell} ; t\right),
\end{aligned}
$$

where

$$
K(x ; t)=1-t\left(x_{1}+\cdots+x_{\ell}+\bar{x}_{1}+\cdots+\bar{x}_{\ell}\right) .
$$

Let us multiply the equation by

$$
M(x):=x_{2} x_{3}^{2} \cdots x_{\ell}^{\ell-1}\left(1-x_{1}\right) \cdots\left(1-x_{\ell}\right) .
$$

This gives

$$
\begin{aligned}
& K(x ; t) M(x) \bar{G}(x ; t) \\
& =M(x)+t x_{2} x_{3}^{2} \cdots x_{\ell}^{\ell-1}\left(1-\bar{x}_{1}\right)\left(1-x_{1}\right) \prod_{j=2}^{\ell}\left(1-x_{j}\right) \bar{G}\left(0, x_{2}, \ldots, x_{\ell} ; t\right) \\
& \quad-t \prod_{j=1}^{\ell}\left(1-x_{j}\right) \sum_{j=2}^{\ell} x_{2} \cdots x_{j-1}^{j-2} x_{j}^{j-2} x_{j+1}^{j} \cdots x_{\ell}^{\ell-1} \\
& \quad \times \bar{G}\left(x_{1}, \ldots, x_{j-2}, x_{j-1}+x_{j}, 0, x_{j+1}, \ldots, x_{\ell} ; t\right) .
\end{aligned}
$$

The term involving $\bar{G}\left(0, x_{2}, \ldots, x_{\ell}\right)$ is invariant under the action of the generator $\sigma_{1}$ of $B_{\ell}$. For $j \geq 2$, the $j^{\text {th }}$ term of the sum is invariant under the action of the generator $\sigma_{j}$. Consequently, forming the signed sum of (20) over the hyperoctahedral group $B_{\ell}$ yields the orbit sum (17), where now $K(x ; t)$ and $M(x)$ are respectively given by (18) and (19). 


\subsection{Extraction of $G(1, \ldots, 1 ; t)$}

- Assume $m$ is odd, and consider the orbit sum (17). For every $\sigma \in B_{\ell}$, the term

$$
\begin{aligned}
\sigma(M(x) \bar{G}(x ; t))= & \sigma\left(x _ { 1 } x _ { 2 } ^ { 2 } \cdots x _ { \ell } ^ { \ell } G \left(\frac{1}{1-t\left(x_{1}+\cdots+x_{\ell}\right)}, \frac{1}{1-t\left(x_{2}+\cdots+x_{\ell}\right)},\right.\right. \\
& \left.\left.\ldots, \frac{1}{1-t x_{\ell}} ; t\right)\right)
\end{aligned}
$$

is a power series in $t$ with coefficients in $\mathbb{Q}\left[x_{1}, \ldots, x_{\ell}, \bar{x}_{1}, \ldots, \bar{x}_{\ell}\right]$. We will prove that the coefficient of $x_{1} \cdots x_{\ell}^{\ell}$ in (17) reduces to $\bar{G}(0, \ldots, 0 ; t)=G(1, \ldots, 1 ; t)$, which is the (ordinary) length generating function of involutions avoiding $(m+1) m \cdots 21$.

First, if $\sigma$ has some signed elements, all monomials in the $x_{i}$ 's occurring in $\sigma(M(x) \bar{G}(x ; t))$ have at least one negative exponent. Hence $\sigma(M(x) \bar{G}(x ; t))$ does not contribute to the coefficient of $x_{1} \cdots x_{\ell}^{\ell}$.

If $\sigma$ is not signed, it is a mere permutation of the $x_{i}$ 's. Each monomial occurring in $\sigma(M(x) \bar{G}(x ; t))$ is of the form $x_{1}^{e_{1}} \cdots x_{\ell}^{e_{\ell}}$, where the $e_{i}$ 's are positive. However, monomials with $e_{1}=1$ only occur if $\sigma(1)=1$ (because of the factor $\left.M(x)=x_{1} x_{2}^{2} \cdots x_{\ell}^{\ell}\right)$. But then, if we also want $e_{2}=2$, the only permutations $\sigma$ that contribute are those that satisfy $\sigma(2)=2$. Iterating this observation, we see that the only permutation $\sigma$ that contributes to the coefficient of $x_{1} x_{2}^{2} \cdots x_{\ell}^{\ell}$ is the identity. Moreover, its contribution is clearly $\bar{G}(0, \ldots, 0 ; t)=G(1, \ldots, 1 ; t)$.

Let us state this as a proposition, in which we have also made explicit the righthand side of the orbit sum.

Proposition 9 If $m=2 \ell+1$, the ordinary generating function of involutions avoiding $(m+1) m \cdots 21$ is the coefficient of $x_{1} x_{2}^{2} \cdots x_{\ell}^{\ell}$ in a rational function:

$$
G_{m}(t):=\sum_{\tau \in \mathfrak{I}^{(m)}} t^{|\tau|}=\left[x_{1} x_{2}^{2} \cdots x_{\ell}^{\ell}\right] \frac{\operatorname{det}\left(x_{j}^{i}-\bar{x}_{j}^{i}\right)_{1 \leq i, j \leq \ell}}{1-t\left(1+x_{1}+\cdots+x_{\ell}+\bar{x}_{1}+\cdots+\bar{x}_{\ell}\right)} .
$$

Equivalently, the exponential generating function of these involutions is

$$
G_{m}^{(e)}(t):=\sum_{\tau \in \mathfrak{I}^{(m)}} \frac{t^{|\tau|}}{|\tau| !}=e^{t}\left[x_{1} x_{2}^{2} \cdots x_{\ell}^{\ell}\right] \operatorname{det}\left(\left(x_{j}^{i}-\bar{x}_{j}^{i}\right) e^{t\left(x_{j}+\bar{x}_{j}\right)}\right)_{1 \leq i, j \leq \ell} .
$$

Proof We have just argued that $G_{m}(t)$ is the coefficient of $x_{1} x_{2}^{2} \cdots x_{\ell}^{\ell}$ in the righthand side of (17). It remains to prove that

$$
\sum_{\sigma \in B_{\ell}} \varepsilon(\sigma) \sigma\left(x_{1}^{1} \cdots x_{\ell}^{\ell}\right)=\operatorname{det}\left(x_{j}^{i}-\bar{x}_{j}^{i}\right)_{1 \leq i, j \leq \ell} .
$$

This is easily proved if we consider that $\sigma$ first replaces some $x_{i}$ 's by their reciprocals, and then permutes the $x_{i}$ 's. More precisely, there is a bijection between $B_{\ell}$ and $\mathfrak{S}_{\ell} \times$ $\mathbb{Z}_{2}^{\ell}$, sending $\sigma$ to $\left(\pi, e_{1}, \ldots, e_{\ell}\right)$, with $\pi \in \mathfrak{S}_{\ell}$ and $e_{i} \in\{-1,1\}$, such that

$$
\sigma\left(P\left(x_{1}, \ldots, x_{\ell}\right)\right)=\pi\left(P\left(x_{1}^{e_{1}}, \ldots, x_{\ell}^{e_{\ell}}\right)\right) \text { and } \varepsilon(\sigma)=\varepsilon(\pi)(-1)^{\sharp\left\{i: e_{i}=-1\right\}} \text {. }
$$


Thus

$$
\begin{aligned}
\sum_{\sigma \in B_{\ell}} \varepsilon(\sigma) \sigma\left(x_{1}^{1} \cdots x_{\ell}^{\ell}\right) & =\sum_{\pi \in \mathfrak{S}_{\ell}} \varepsilon(\pi) \sum_{e_{1}, \ldots, e_{\ell} \in\{-1,1\}}(-1)^{\sharp\left\{i: e_{i}=-1\right\}} x_{\pi(1)}^{e_{1}} x_{\pi(2)}^{2 e_{2}} \cdots x_{\pi(\ell)}^{\ell e_{\ell}} \\
& =\sum_{\pi \in \mathfrak{S}_{\ell}} \varepsilon(\pi) \prod_{i=1}^{\ell}\left(x_{\pi(i)}^{i}-\bar{x}_{\pi(i)}^{i}\right) \\
& =\operatorname{det}\left(x_{j}^{i}-\bar{x}_{j}^{i}\right)_{1 \leq i, j \leq \ell} .
\end{aligned}
$$

This gives the expression of $G_{m}(t)$. We then convert it into an expression for the exponential generating function $G_{m}^{(e)}(t)$ by observing that the ordinary generating function $1 /(1-a t)=\sum_{n} t^{n} a^{n}$ corresponds to the exponential generating function $\sum_{n} t^{n} a^{n} / n !=\exp (a t)$.

Remark The determinant occurring in the proposition can be evaluated in closed form (see, e.g., [24, Lemma 2]):

$$
\operatorname{det}\left(x_{j}^{i}-\bar{x}_{j}^{i}\right)_{1 \leq i, j \leq \ell}=\left(x_{1} \cdots x_{\ell}\right)^{-\ell} \prod_{i=1}^{\ell}\left(x_{i}^{2}-1\right) \prod_{1 \leq i<j \leq \ell}\left(\left(x_{i}-x_{j}\right)\left(1-x_{i} x_{j}\right)\right)
$$

but this is not needed here.

- Assume now that $m=2 \ell$ is even. The identity (17) still holds, with $K(x ; t)$ and $M(x)$ given by (18) and (19). Based on the study of the odd case, it would be tempting to extract the coefficient of $x_{2} \cdots x_{\ell}^{\ell-1}$ in this identity. However, this will not give $\bar{G}(0, \ldots, 0 ; t)$, as both $\sigma=\mathrm{id}$ and $\sigma=\sigma_{1}$ (the generator of $B_{\ell}$ that replaces $x_{1}$ by $\left.\bar{x}_{1}\right)$ contribute to this coefficient. But we note that each term in the equation is a multiple of $P(x):=\prod_{i=1}^{\ell}\left(1-x_{i}\right)$. Hence we will first divide by $P(x)$. Let us study the action of $\sigma \in B_{\ell}$ on $P(x)$, with $\sigma$ described as in (21). We have:

$$
\begin{aligned}
\sigma(P(x)) & =\pi\left(\left(1-x_{1}^{e_{1}}\right) \cdots\left(1-x_{\ell}^{e_{\ell}}\right)\right)=\pi\left(P(x) \prod_{i: e_{i}=-1}\left(-\bar{x}_{i}\right)\right) \\
& =(-1)^{\sharp\left\{i: e_{i}=-1\right\}} P(x) \prod_{i: e_{i}=-1} \bar{x}_{\pi(i)} .
\end{aligned}
$$

Hence, denoting $e=\left(e_{1}, \ldots, e_{\ell}\right), x^{e}=\left(x_{1}^{e_{1}}, \ldots, x_{\ell}^{e_{\ell}}\right)$ and $N(x)=x_{2} \cdots x_{\ell}^{\ell-1}$, dividing (17) by $P(x)$ gives

$$
\begin{aligned}
& \sum_{\substack{\pi \in \mathfrak{S}_{\ell} \\
e \in\{-1,1\}^{\ell}}} \varepsilon(\pi) \pi\left(N\left(x^{e}\right) \bar{G}\left(x^{e} ; t\right) \prod_{i: e_{i}=-1} \bar{x}_{i}\right) \\
& =\frac{1}{K(x ; t)}\left(\sum_{\substack{\pi \in \mathfrak{S}_{\ell} \\
e \in\{-1,1\}^{\ell}}} \varepsilon(\pi) \pi\left(N\left(x^{e}\right) \prod_{i: e_{i}=-1} \bar{x}_{i}\right)\right) .
\end{aligned}
$$


Let us now extract from the left-hand side the coefficient of $x_{2} \cdots x_{\ell}^{\ell-1}$. The argument is similar to the odd case. If $e \neq(1, \ldots, 1)$, each monomial occurring in $N\left(x^{e}\right) \bar{G}\left(x^{e} ; t\right) \prod_{i: e_{i}=-1} \bar{x}_{i}$ contains a negative exponent, and thus cannot contribute. Now for $e=(1, \ldots, 1)$, the term $\pi(N(x) \bar{G}(x ; t))$ only contributes if $\pi=\mathrm{id}$, and then its contribution is $\bar{G}(0, \ldots, 0 ; t)$, the length generating function of involutions avoiding $(m+1) m \cdots 21$. We obtain the following counterpart of Proposition 9.

Proposition 10 If $m=2 \ell$, the ordinary generating function of involutions avoiding $(m+1) m \cdots 21$ is the coefficient of $x_{1}^{0} x_{2}^{1} \cdots x_{\ell}^{\ell-1}$ in a rational function:

$$
G_{m}(t):=\sum_{\tau \in \mathfrak{I}^{(m)}} t^{|\tau|}=\left[x_{1}^{0} x_{2}^{1} \cdots x_{\ell}^{\ell-1}\right] \frac{\operatorname{det}\left(x_{j}^{i-1}+\bar{x}_{j}^{i}\right)_{1 \leq i, j \leq \ell}}{1-t\left(x_{1}+\cdots+x_{\ell}+\bar{x}_{1}+\cdots+\bar{x}_{\ell}\right)} .
$$

Equivalently, the exponential generating function of these involutions is

$$
G_{m}^{(e)}(t):=\sum_{\tau \in \mathfrak{I}^{(m)}} \frac{t^{|\tau|}}{|\tau| !}=\left[x_{1}^{0} x_{2}^{1} \cdots x_{\ell}^{\ell-1}\right] \operatorname{det}\left(\left(x_{j}^{i-1}+\bar{x}_{j}^{i}\right) e^{t\left(x_{j}+\bar{x}_{j}\right)}\right)_{1 \leq i, j \leq \ell} .
$$

Proof We have just argued that $G_{m}(t)$ is the coefficient of $x_{2}^{1} \cdots x_{\ell}^{\ell-1}$ in the righthand side of (22). It remains to evaluate the numerator in the right-hand side:

$$
\begin{aligned}
\sum_{\substack{\pi \in \mathfrak{S}_{\ell} \\
e \in\{-1,1\}^{\ell}}} \varepsilon(\pi) \pi\left(N\left(x^{e}\right) \prod_{i: e_{i}=-1} \bar{x}_{i}\right) & =\sum_{\pi \in \mathfrak{S}_{\ell}} \varepsilon(\pi) \pi\left(\sum_{\ell \in\{-1,1\}^{\ell}} \prod_{i=1}^{\ell} x_{i}^{\left.(i-1) e_{i}-\chi_{e_{i}=-1}\right)}\right. \\
& =\sum_{\pi \in \mathfrak{S}_{\ell}} \varepsilon(\pi) \pi\left(\prod_{i=1}^{\ell}\left(x_{i}^{i-1}+\bar{x}_{i}^{i}\right)\right) \\
& =\operatorname{det}\left(x_{j}^{i-1}+\bar{x}_{j}^{i}\right) .
\end{aligned}
$$

This gives the expression of $G_{m}(t)$. Taking the corresponding exponential generating function gives $G_{m}^{(e)}(t)$.

Remark Again, the determinant occurring in the proposition can be evaluated in closed form [24, (2.6)], but this is not needed here.

\subsection{Determinantal expression of the series}

- Let us assume that $m$ is odd, and return to Proposition 9. Taking the exponential generating function rather than the ordinary one makes the extraction of the coefficient of $x_{1} \cdots x_{\ell}^{\ell}$ an elementary task, as all variables $x_{j}$ decouple. The series $I_{i}$ defined by (1) arise naturally from

$$
\left[x^{i}\right] e^{t(x+\bar{x})}=I_{i} .
$$


We have:

$$
\begin{aligned}
G_{m}^{(e)}(t) & =e^{t} \sum_{\pi \in \mathfrak{S}_{\ell}} \varepsilon(\pi) \prod_{i=1}^{\ell}\left[x_{i}^{i}\right]\left(\left(x_{i}^{\pi(i)}-\bar{x}_{i}^{\pi(i)}\right) e^{t\left(x_{i}+\bar{x}_{i}\right)}\right) \\
& =e^{t} \sum_{\pi \in \mathfrak{S}_{\ell}} \varepsilon(\pi) \prod_{i=1}^{\ell}\left(I_{i-\pi(i)}-I_{i+\pi(i)}\right) \\
& =e^{t} \operatorname{det}\left(I_{i-j}-I_{i+j}\right)_{1 \leq i, j \leq \ell} .
\end{aligned}
$$

We have thus recovered the first part of Theorem 2.

- If $m$ is even, we start from Proposition 10. Again, the variables $x_{j}$ decouple in the exponential generating function:

$$
\begin{aligned}
G_{m}^{(e)}(t) & =\sum_{\pi \in \mathfrak{S}_{\ell}} \varepsilon(\pi) \prod_{i=1}^{\ell}\left[x_{i}^{i-1}\right]\left(\left(x_{i}^{\pi(i)-1}+\bar{x}_{i}^{\pi(i)}\right) e^{t\left(x_{i}+\bar{x}_{i}\right)}\right) \\
& =\sum_{\pi \in \mathfrak{S}_{\ell}} \varepsilon(\pi) \prod_{i=1}^{\ell}\left(I_{i-\pi(i)}+I_{i+\pi(i)-1}\right) \\
& =\operatorname{det}\left(I_{i-j}+I_{i+j-1}\right)_{1 \leq i, j \leq \ell}
\end{aligned}
$$

We have thus recovered the second part of Theorem 2 .

Remark The determinantal expression of $G_{m}^{(e)}$ implies that this series is D-finite, that is, satisfies a linear differential equation with polynomial coefficients. However, this follows as well from the constant term expressions of Propositions 9 and 10 using the closure properties of D-finite series [27, 28].

\subsection{The number of fixed points}

We now enrich our results by taking into account the number of fixed points, thereby recovering Theorem 3. Recall from Proposition 6 that the label of the involution obtained by inserting $n+1$ as a fixed point in $\tau \in \mathfrak{I}_{n}^{(m)}$ is $\left(a_{1}+1, a_{2}+1, \ldots, a_{\ell}+1\right)$ if $m$ is odd, $\left(1, a_{2}+1, \ldots, a_{\ell}+1\right)$ otherwise. Hence, if we keep track of the number of fixed points by a new variable $s$, the functional equation of Proposition 7 becomes

$$
\begin{aligned}
& G(v ; t, s) \\
& \quad=v_{1}+s t v_{1} G(v ; t, s) \chi_{m \equiv 1}+s t v_{1} G\left(v_{2}, v_{2}, v_{3}, \ldots, v_{\ell} ; t, s\right) \chi_{m \equiv 0} \\
& \quad+t^{2} v_{1} \sum_{j=1}^{\ell} v_{j} v_{j+1} \frac{G(v ; t, s)-G\left(v_{1}, \ldots, v_{j-1}, v_{j+1}, v_{j+1}, v_{j+2}, \ldots, v_{\ell} ; t, s\right)}{v_{j}-v_{j+1}} .
\end{aligned}
$$


The series $G(1, \ldots, 1 ; t, s)$ counts involutions of $\mathfrak{I}^{(m)}$ by their length and number of fixed points. The change of variables (13) now gives

$$
\begin{aligned}
K(x ; t, s) \bar{G}(x ; t, s)= & 1+\operatorname{st} \bar{G}\left(0, x_{2}, \ldots, x_{\ell} ; t, s\right) \chi_{m \equiv 0} \\
& -t \sum_{j=1}^{\ell} \bar{x}_{j} \bar{G}\left(x_{1}, \ldots, x_{j-2}, x_{j-1}+x_{j}, 0, x_{j+1}, \ldots, x_{\ell} ; t, s\right),
\end{aligned}
$$

where

$$
K(x ; t, s)=1-t\left(x_{1}+\cdots+x_{\ell}\right)-s t \chi_{m \equiv 1}-t\left(\bar{x}_{1}+\cdots+\bar{x}_{\ell}\right),
$$

and

$$
\begin{aligned}
\bar{G}(x ; t, s) & \equiv \bar{G}\left(x_{1}, \ldots, x_{\ell} ; t\right) \\
& =G\left(\frac{1}{1-t\left(x_{1}+\cdots+x_{\ell}\right)}, \frac{1}{1-t\left(x_{2}+\cdots+x_{\ell}\right)}, \ldots, \frac{1}{1-t x_{\ell}} ; t, s\right) .
\end{aligned}
$$

- If $m$ is odd, the argument of Sects. 4.2, 4.3, 4.4, applies verbatim. The only difference is that the term $t$ occurring in the kernel is replaced by $s t$. This gives at once the first part of Theorem 3, in the form (3).

- If $m$ is even, the equation reads

$$
\begin{aligned}
K(x ; t) \bar{G}(x ; t, s)= & 1+t\left(s-\bar{x}_{1}\right) \bar{G}\left(0, x_{2}, \ldots, x_{\ell} ; t, s\right) \\
& -t \sum_{j=2}^{\ell} \bar{x}_{j} \bar{G}\left(x_{1}, \ldots, x_{j-2}, x_{j-1}+x_{j}, 0, x_{j+1}, \ldots, x_{\ell} ; t, s\right)
\end{aligned}
$$

with $K(x ; t)=1-t\left(x_{1}+\cdots+x_{\ell}+\bar{x}_{1}+\cdots+\bar{x}_{\ell}\right)$. We multiply it by

$$
M(x ; s):=x_{2} x_{3}^{2} \cdots x_{\ell}^{\ell-1}\left(s-x_{1}\right) \cdots\left(s-x_{\ell}\right),
$$

and then argue as in Sect. 4.2 to conclude that

$$
\sum_{\sigma \in B_{\ell}} \varepsilon(\sigma) \sigma(M(x ; s) \bar{G}(x ; t, s))=\frac{\sum_{\sigma \in B_{\ell}} \varepsilon(\sigma) \sigma(M(x ; s))}{K(x ; t)},
$$

with the above values of $K(x ; t)$ and $M(x ; s)$.

Now we cannot follow exactly the argument of Sect. 4.3, because $\sigma(M(x ; s))$ does not differ from $M(x ; s)$ by a monomial. So it does not help to divide the equation by $\left(s-x_{1}\right) \cdots\left(s-x_{\ell}\right)$. Instead, let us leave the equation as it is, and extract all terms of the form $x_{1}^{a} x_{2}^{1} \cdots x_{\ell}^{\ell-1}$ with $a \geq 0$. More precisely, for a series $F\left(x_{1}, \ldots, x_{\ell} ; t, s\right)$ in $\mathbb{Q}\left[x_{1}, \ldots, x_{\ell}, s\right][[t]]$, let us denote

$$
\left[x_{1}^{\geq 0} x_{2}^{1} \cdots x_{\ell}^{\ell-1}\right] F\left(x_{1}, \ldots, x_{\ell} ; t, s\right):=\sum_{a \geq 0} x_{1}^{a}\left[x_{1}^{a} x_{2}^{1} \cdots x_{\ell}^{\ell-1}\right] F\left(x_{1}, \ldots, x_{\ell} ; t, s\right) .
$$


Consider the term

$$
\sigma(M(x ; s) \bar{G}(x ; t, s))=\sigma\left(x_{2} x_{3}^{2} \cdots x_{\ell}^{\ell-1}\left(s-x_{1}\right) \cdots\left(s-x_{\ell}\right) \bar{G}(x ; t, s)\right) .
$$

Let us decouple in $\sigma$ the sign changes $e_{1}, \ldots, e_{\ell}$ and the permutation $\pi$ of the $x_{i}$ 's, as in (21). We wish to determine $\left[x_{1}^{\geq 0} x_{2}^{1} \cdots x_{\ell}^{\ell-1}\right] \sigma(M(x ; s) \bar{G}(x ; t, s))$.

- If one of the $e_{i}$ 's, for $i \geq 2$, is -1 , then all monomials occurring in $\sigma(M(x ; s) \bar{G}(x ; t, s))$ involve a negative exponent and thus do not contribute.

- If $e_{1}=-1$ while $e_{i}=1$ for $i \geq 2$, the only way to obtain a non-zero contribution of $\sigma(M(x ; s) \bar{G}(x ; t, s))$ is to take $\pi=\mathrm{id}$, and the contribution is then

$$
s^{\ell} \bar{G}(0, \ldots, 0 ; t, s) \text {. }
$$

- If $\sigma=\pi \in \mathfrak{S}_{\ell}$, the contribution is

$$
\left(s-x_{1}\right)\left[x_{1}^{\geq 0} x_{2}^{1} \cdots x_{\ell}^{\ell-1}\right]\left(\left(s-x_{2}\right) \cdots\left(s-x_{\ell}\right) \pi\left(x_{2}^{1} \cdots x_{\ell}^{\ell-1} \bar{G}(x ; t, s)\right)\right) .
$$

We note that this is a multiple of $\left(s-x_{1}\right)$.

Hence, the result of our coefficient extraction on (23) is

$$
\begin{aligned}
& -s^{\ell} \bar{G}(0, \ldots, 0 ; t, s)+\left(s-x_{1}\right) \sum_{\pi \in \mathfrak{S}_{\ell}} \varepsilon(\pi)\left[x_{1}^{\geq 0} x_{2}^{1} \cdots x_{\ell}^{\ell-1}\right]\left(\left(s-x_{2}\right) \cdots\left(s-x_{\ell}\right)\right. \\
& \left.\quad \times \pi\left(x_{2}^{1} \cdots x_{\ell}^{\ell-1} \bar{G}(x ; t, s)\right)\right) \\
& =\left[x_{1}^{\geq 0} x_{2}^{1} \cdots x_{\ell}^{\ell-1}\right] \frac{\sum_{\sigma \in B_{\ell}} \varepsilon(\sigma) \sigma(M(x ; s))}{K(x ; t)} .
\end{aligned}
$$

Let us specialize this to $x_{1}=s$ :

$$
-s^{\ell} \bar{G}(0, \ldots, 0 ; t, s)=\left.\left(\left[x_{1}^{\geq 0} x_{2}^{1} \cdots x_{\ell}^{\ell-1}\right] \frac{\sum_{\sigma \in B_{\ell}} \varepsilon(\sigma) \sigma(M(x ; s))}{K(x ; t)}\right)\right|_{x_{1} \mapsto s} .
$$

The kernel $K(x ; t)$ is independent of $s$. But this is also the case of

$$
\begin{aligned}
\sum_{\sigma \in B_{\ell}} \varepsilon(\sigma) \sigma(M(x ; s)) & =\sum_{\pi \in \mathfrak{S}_{\ell}} \varepsilon(\pi) \pi\left(\prod_{i=1}^{\ell}\left(\left(s-x_{i}\right) x_{i}^{i-1}-\left(s-\bar{x}_{i}\right) \bar{x}_{i}^{i-1}\right)\right) \\
& =\operatorname{det}\left(s\left(x_{j}^{i-1}-\bar{x}_{j}^{i-1}\right)-x_{j}^{i}+\bar{x}_{j}^{i}\right)_{1 \leq i, j \leq \ell} \\
& =\operatorname{det}\left(-x_{j}^{i}+\bar{x}_{j}^{i}\right)_{1 \leq i, j \leq \ell}
\end{aligned}
$$

as is seen by taking linear combinations of rows. We have thus obtained the following counterpart of Proposition 10.

Proposition 11 If $m=2 \ell$, the ordinary generating function of involutions avoiding $(m+1) m \cdots 21$, counted by the length and number of fixed points, is, with the nota- 
tion (24):

$$
\begin{aligned}
G_{m}\left(t, x_{1}\right) & :=\sum_{\tau \in \mathfrak{I}^{(m)}} t^{|\tau|} x_{1}^{f(\tau)} \\
& =-\frac{1}{x_{1}^{\ell}}\left[x_{1}^{\geq 0} x_{2}^{1} \cdots x_{\ell}^{\ell-1}\right] \frac{\operatorname{det}\left(\bar{x}_{j}^{i}-x_{j}^{i}\right)_{1 \leq i, j \leq \ell}}{1-t\left(x_{1}+\cdots+x_{\ell}+\bar{x}_{1}+\cdots+\bar{x}_{\ell}\right)} .
\end{aligned}
$$

Equivalently, the exponential generating function of these involutions is

$$
\begin{aligned}
G_{m}^{(e)}\left(t, x_{1}\right) & :=\sum_{\tau \in \mathfrak{I}^{(m)}} \frac{t^{|\tau|}}{|\tau| !} x_{1}^{f(\tau)} \\
& =-\frac{1}{x_{1}^{\ell}}\left[x_{1}^{\geq 0} x_{2}^{1} \cdots x_{\ell}^{\ell-1}\right] \operatorname{det}\left(\left(\bar{x}_{j}^{i}-x_{j}^{i}\right) e^{t\left(x_{j}+\bar{x}_{j}\right)}\right)_{1 \leq i, j \leq \ell} .
\end{aligned}
$$

We can now perform the coefficient extraction explicitly in the expression of $G_{m}^{(e)}\left(t, x_{1}\right)$ :

$$
\begin{aligned}
G_{m}^{(e)}\left(t, x_{1}\right)= & -\frac{1}{x_{1}^{\ell}} \sum_{\pi \in \mathfrak{S}_{\ell}} \varepsilon(\pi)\left[x_{1}^{\geq 0}\right]\left(\left(\bar{x}_{1}^{\pi(1)}-x_{1}^{\pi(1)}\right) e^{t\left(x_{1}+\bar{x}_{1}\right)}\right) \\
& \times \prod_{i=2}^{\ell}\left[x_{i}^{i-1}\right]\left(\left(\bar{x}_{i}^{\pi(i)}-x_{i}^{\pi(i)}\right) e^{t\left(x_{i}+\bar{x}_{i}\right)}\right) \\
= & -\frac{1}{x_{1}^{\ell}} \sum_{\pi \in \mathfrak{S}_{\ell}} \varepsilon(\pi) \sum_{k \geq 0} x_{1}^{k}\left(I_{k+\pi(1)}-I_{k-\pi(1)}\right) \prod_{i=2}^{\ell}\left(I_{i+\pi(i)-1}-I_{i-\pi(i)-1}\right) \\
= & \sum_{k \geq 0} x_{1}^{k-\ell} \operatorname{det}\left(\begin{array}{c}
\left(I_{k-j}-I_{k+j}\right)_{1 \leq j \leq \ell} \\
\left(I_{i+j-1}-I_{i-j-1}\right)_{2 \leq i \leq \ell, 1 \leq j \leq \ell}
\end{array}\right) .
\end{aligned}
$$

Upon extracting the coefficient of $x_{1}^{p}$, this gives the second part of Theorem 3 .

\section{Permutations with no long ascending subsequence}

We now want to derive from the functional equation of Proposition 5 the Bessel generating function of permutations avoiding $12 \cdots m(m+1)$, given in Theorem 1 . We follow the same steps as in the case of involutions, but the coefficient extraction is more delicate.

5.1 Invariance properties of the kernel

The kernel of the equation of Proposition 5, that is, the coefficient of $F(v ; t)$, reads

$$
1-t v_{1}-t \sum_{j=2}^{m} \frac{v_{j-1} v_{j}}{v_{j-1}-v_{j}}
$$


Its invariance properties appear clearly if we set

$$
v_{j}=\frac{1}{x_{1}+\cdots+x_{j}} .
$$

Indeed, the kernel then becomes

$$
K(x ; t):=1-t\left(\bar{x}_{1}+\cdots+\bar{x}_{m}\right),
$$

with $\bar{x}_{i}=1 / x_{i}$, and is invariant under the action of the symmetric group $\mathfrak{S}_{m}$, seen as a group of transformations of Laurent polynomials in the $x_{i}$. This group is generated by $m-1$ elements of order 2 , denoted $\sigma_{1}, \ldots, \sigma_{m-1}$ :

$$
\sigma_{j}\left(P\left(x_{1}, \ldots, x_{m}\right)\right)=P\left(x_{1}, \ldots, x_{j-1}, x_{j+1}, x_{j}, x_{j+2}, \ldots, x_{m}\right) .
$$

The functional equation now reads

$$
K(x ; t) \bar{F}(x ; t)=1-t \sum_{j=1}^{m-1} \bar{x}_{j+1} \bar{F}\left(x_{1}, \ldots, x_{j-1}, x_{j}+x_{j+1}, 0, x_{j+2}, \ldots, x_{m} ; t\right),
$$

with

$$
\bar{F}(x ; t) \equiv \bar{F}\left(x_{1}, \ldots, x_{m} ; t\right)=F\left(\frac{1}{x_{1}}, \frac{1}{x_{1}+x_{2}}, \ldots, \frac{1}{x_{1}+\cdots+x_{m}} ; t\right) .
$$

\subsection{Orbit sum}

Let us multiply the equation by

$$
M(x)=x_{1}^{0} x_{2}^{1} \cdots x_{m}^{m-1} .
$$

This gives:

$$
\begin{aligned}
& K(x ; t) M(x) \bar{F}(x ; t) \\
& =M(x)-t \sum_{j=1}^{m-1} x_{1}^{0} \cdots x_{j}^{j-1} x_{j+1}^{j-1} x_{j+2}^{j+1} \cdots x_{m}^{m-1} \\
& \quad \times \bar{F}\left(x_{1}, \ldots, x_{j-1}, x_{j}+x_{j+1}, 0, x_{j+2}, \ldots, x_{m} ; t\right) .
\end{aligned}
$$

The $j^{\text {th }}$ term of the sum is invariant under the action of the generator $\sigma_{j}$ (which exchanges $x_{j}$ and $x_{j+1}$ ). Consequently, forming the signed sum of (28) over the symmetric group $\mathfrak{S}_{m}$ gives the following orbit sum:

$$
\sum_{\sigma \in \mathfrak{S}_{m}} \varepsilon(\sigma) \sigma(K(x ; t) M(x) \bar{F}(x ; t))=\sum_{\sigma \in \mathfrak{S}_{m}} \varepsilon(\sigma) \sigma(M(x)),
$$


or, given that $K(x ; t)$ is $\mathfrak{S}_{m}$-invariant,

$$
\sum_{\sigma \in \mathfrak{S}_{m}} \varepsilon(\sigma) \sigma(M(x) \bar{F}(x ; t))=\frac{\sum_{\sigma \in \mathfrak{S}_{m}} \varepsilon(\sigma) \sigma(M(x))}{K(x ; t)}=\frac{\operatorname{det}\left(x_{j}^{i-1}\right)_{1 \leq i, j \leq m}}{K(x ; t)},
$$

where $K(x ; t)$ is given by (25) and $M(x)$ by (27).

5.3 Extraction of $F(1, \ldots, 1 ; t)$

For $1 \leq j \leq m$, let us now denote $z_{j}=x_{1}+\cdots+x_{j}$. Equivalently, $x_{j}=z_{j}-z_{j-1}$ with $z_{0}=0$. All series occurring in the orbit sum (29) become series in $t$ with coefficients in $\mathbb{Q}\left(z_{1}, \ldots, z_{m}\right)$. In particular,

$$
\bar{F}(x ; t)=F\left(\frac{1}{z_{1}}, \frac{1}{z_{2}}, \ldots, \frac{1}{z_{m}} ; t\right)
$$

has coefficients which are Laurent polynomials in the $z_{j}$ 's. This is not the case for all terms in (29). For instance, if $\sigma$ is the 2-cycle $(1,2)$,

$\sigma(\bar{F}(x ; t))=F\left(\frac{1}{x_{2}}, \frac{1}{x_{1}+x_{2}}, \ldots, \frac{1}{x_{1}+\cdots+x_{m}} ; t\right)=F\left(\frac{1}{z_{2}-z_{1}}, \frac{1}{z_{2}}, \ldots, \frac{1}{z_{m}} ; t\right)$

involves coefficients which are not Laurent polynomials in the $z_{j}$ 's. In order to perform our extraction, we will expand all rational functions of the $z_{j}$ 's as (iterated) Laurent series, by expanding first in $z_{1}$, then in $z_{2}$, and so on. For instance, the expansion of $1 /\left(x_{1}+x_{3}+x_{4}\right)$ reads

$$
\begin{aligned}
\frac{1}{x_{1}+x_{3}+x_{4}} & =\frac{1}{z_{4}-z_{2}+z_{1}}=\sum_{e_{1} \geq 0} \frac{\left(-z_{1}\right)^{e_{1}}}{\left(z_{4}-z_{2}\right)^{e_{1}+1}} \\
& =\sum_{e_{1} \geq 0, e_{2} \geq 0}\left(\begin{array}{c}
e_{1}+e_{2} \\
e_{1}
\end{array}\right) \frac{\left(-z_{1}\right)^{e_{1}} z_{2}^{e_{2}}}{z_{4}^{1+e_{1}+e_{2}}} .
\end{aligned}
$$

In other words, the coefficients of our series in $t$ now lie in the ring of iterated Laurent series in $z_{1}, \ldots, z_{m}$, which is defined inductively as follows:

- if $m=1$, it coincides with the ring of Laurent series ${ }^{1}$ in $z_{1}$ (with rational coefficients),

- if $m>1$, it is the ring of Laurent series in $z_{1}$ whose coefficients are iterated Laurent series in $z_{2}, \ldots, z_{m}$.

It follows from this definition that an iterated Laurent series in the $z_{j}$ 's only contains finitely many non-positive monomials, that is, monomials $z_{1}^{e_{1}} \cdots z_{m}^{e_{m}}$ with $e_{j} \leq 0$ for all $j$. This allows us to define below a linear operator $\Lambda$, which extracts from an iterated Laurent series some coefficients associated with non-positive monomials and adds them up.

\footnotetext{
${ }^{1}$ Recall that our Laurent series only involve finitely many negative exponents.
} 
Definition 12 Let $\Lambda$ be the linear operator defined on iterated Laurent series in $z_{1}, \ldots, z_{m}$ by the following action on monomials:

$$
\Lambda\left(z_{1}^{e_{1}} \cdots z_{m}^{e_{m}}\right)= \begin{cases}1, & \text { if } e_{1} \leq 0, \ldots, e_{m} \leq 0 \text { and } e_{j}=0 \Rightarrow e_{j+1}=\cdots=e_{m}=0 \\ 0, & \text { otherwise }\end{cases}
$$

Remark The action of $\Lambda$ can also be described as the extraction of a constant term: for any iterated Laurent series $F\left(z_{1}, \ldots, z_{m}\right)$,

$$
\Lambda\left(F\left(z_{1}, \ldots, z_{m}\right)\right)=\left[z_{1}^{0} \cdots z_{m}^{0}\right]\left(F\left(z_{1}, \ldots, z_{m}\right) \sum_{i=0}^{m} \prod_{j=1}^{i} \frac{z_{j}}{1-z_{j}}\right) .
$$

This operator has been designed to extract from (29) the series $F(1, \ldots, 1 ; t)$ in which we are interested. The following proposition is thus the counterpart of Propositions 9 and 10 .

Proposition 13 The ordinary generating function of permutations avoiding $12 \cdots m(m+1)$ is obtained by applying $\Lambda$ to a rational function:

$$
F_{m}(t):=\sum_{\tau \in \mathfrak{S}^{(m)}} t^{|\tau|}=\Lambda\left(\frac{\operatorname{det}\left(x_{j}^{i-j}\right)_{1 \leq i, j \leq m}}{1-t\left(\bar{x}_{1}+\cdots+\bar{x}_{m}\right)}\right)
$$

with $x_{j}=z_{j}-z_{j-1}$ and $z_{0}=0$.

Equivalently, the exponential generating function of these permutations is

$$
F_{m}^{(e)}(t):=\sum_{\tau \in \mathfrak{S}^{(m)}} \frac{t^{|\tau|}}{|\tau| !}=\Lambda\left(\operatorname{det}\left(x_{j}^{i-j} e^{t \bar{x}_{j}}\right)_{1 \leq i, j \leq m}\right)
$$

\section{Remarks}

1. The fact that the action of $\Lambda$ can be described as a constant term extraction, combined with closure properties of D-finite series [27, 28], implies that the series $F_{m}$ (and $F_{m}^{(e)}$ ) are D-finite. This was first proved by Gessel [16].

2. Again, the determinant is a Vandermonde determinant and can be evaluated in closed form, but this will not be needed.

Proof We will prove that for all $\sigma \in \mathfrak{S}_{m}$,

$$
\Lambda\left(\frac{\sigma(M(x) \bar{F}(x ; t))}{M(x)}\right)= \begin{cases}F(1, \ldots, 1 ; t), & \text { if } \sigma=\mathrm{id} \\ 0, & \text { otherwise }\end{cases}
$$

so that the first result directly follows from (29), after dividing by $M(x)$ and applying $\Lambda$. It is then easily converted into an expression for the exponential generating function. 
Recall the Definition (26) of $\bar{F}(x ; t)$, and that $\mathfrak{S}_{m}$ acts by permuting the $x_{j}$ 's. Also,

$$
F\left(v_{1}, \ldots, v_{m} ; t\right)=\sum_{\tau \in \mathfrak{S}^{(m)}} v_{1}^{a_{2}(\tau)-1} v_{2}^{a_{3}(\tau)-a_{2}(\tau)} \cdots v_{m}^{|\tau|+1-a_{m}(\tau)} t^{|\tau|},
$$

where the labels $a_{i}(\tau)$ are defined by (4). This definition implies that, if $a_{j}(\tau)=$ $a_{j+1}(\tau)$, then $a_{j}(\tau)=a_{j+1}(\tau)=\cdots=a_{m}(\tau)=|\tau|+1$. In other words, the $v$ monomials occurring in $F(v ; t)$ satisfy a property that should be reminiscent of the definition of $\Lambda$ :

$$
F\left(v_{1}, \ldots, v_{m} ; t\right)=\sum_{\left(e_{1}, \ldots, e_{m}\right) \in \mathcal{E}} c\left(e_{1}, \ldots, e_{m}\right) v_{1}^{e_{1}} v_{2}^{e_{2}} \cdots v_{m}^{e_{m}} t^{e_{1}+\cdots+e_{m}},
$$

where

$$
\mathcal{E}=\left\{\left(e_{1}, \ldots, e_{m}\right) \in \mathbb{N}^{m}: e_{j}=0 \Rightarrow e_{j+1}=\cdots=e_{m}=0\right\} .
$$

With this property at hand, we can now address the proof of (31). If $\sigma=\mathrm{id}$,

$$
\Lambda\left(\frac{\sigma(M(x) \bar{F}(x ; t))}{M(x)}\right)=\Lambda\left(F\left(\frac{1}{z_{1}}, \frac{1}{z_{2}}, \ldots, \frac{1}{z_{m}} ; t\right)\right)=F(1, \ldots, 1 ; t)
$$

by definition of $\Lambda$ and (32).

It remains to prove the second part of (31). Let us consider an example, say $m=5$ and $\sigma=13425$. Let $\tau \in \mathfrak{S}_{n}^{(5)}$, and denote $e_{i}=a_{i+1}(\tau)-a_{i}(\tau)$, with $a_{1}(\tau)=1$ and $a_{m+1}(\tau)=|\tau|+1$. Of course, $e_{i} \geq 0$ for all $i$. Up to a factor $t^{|\tau|}$, the contribution of $\tau$ in $\sigma(M(x) \bar{F}(x ; t)) / M(x)$ is

$$
\begin{aligned}
& \frac{1}{x_{2} x_{3}^{2} x_{4}^{3} x_{5}^{4}} \sigma\left(\frac{x_{2} x_{3}^{2} x_{4}^{3} x_{5}^{4}}{x_{1}^{e_{1}}\left(x_{1}+x_{2}\right)^{e_{2}} \cdots\left(x_{1}+\cdots+x_{5}\right)^{e_{5}}}\right) \\
& =\frac{x_{3} x_{4}^{2} x_{2}^{3} x_{5}^{4}}{x_{2} x_{3}^{2} x_{4}^{3} x_{5}^{4} x_{1}^{e_{1}}\left(x_{1}+x_{3}\right)^{e_{2}}\left(x_{1}+x_{3}+x_{4}\right)^{e_{3}}\left(x_{1}+x_{2}+x_{3}+x_{4}\right)^{e_{4}}\left(x_{1}+\cdots+x_{5}\right)^{e_{5}}} \\
& =\frac{\left(z_{2}-z_{1}\right)^{2}}{\left(z_{3}-z_{2}\right)\left(z_{4}-z_{3}\right) z_{1}^{e_{1}}\left(z_{3}-z_{2}+z_{1}\right)^{e_{2}}\left(z_{4}-z_{2}+z_{1}\right)^{e_{3}} z_{4}^{e_{4}} z_{5}^{e_{5}}} .
\end{aligned}
$$

To prepare the Laurent expansion in the variables $z_{i}$, we rewrite this fraction as

$$
\frac{\left(z_{2}-z_{1}\right)^{2}}{z_{1}^{e_{1}} z_{3}^{1+e_{2}} z_{4}^{1+e_{3}+e_{4}} z_{5}^{e_{5}}\left(1-\frac{z_{3}}{z_{4}}\right)\left(1-\frac{z_{2}}{z_{3}}\right)^{1+e_{2}}\left(1-\frac{z_{2}}{z_{4}}\right)^{e_{3}}\left(1+\frac{z_{1}}{z_{3}\left(1-\frac{z_{2}}{z_{3}}\right)}\right)^{e_{2}}\left(1+\frac{z_{1}}{z_{4}\left(1-\frac{z_{2}}{z_{4}}\right)}\right)^{e_{3}}} .
$$

It is now clear that, in each term of the Laurent expansion, $z_{2}$ has a non-negative exponent, while $z_{4}$ has a negative exponent. By definition of $\Lambda$, this implies that

$$
\Lambda\left(\frac{1}{M(x)} \sigma\left(\frac{M(x)}{x_{1}^{e_{1}}\left(x_{1}+x_{2}\right)^{e_{2}} \cdots\left(x_{1}+\cdots+x_{5}\right)^{e_{5}}}\right)\right)=0 .
$$


As this holds for every permutation $\tau \in \mathfrak{S}_{n}^{(5)}$, we have proved that (31) holds for $\sigma=13425$.

Let us say that a series of $\mathbb{Q}\left(x_{1}, \ldots, x_{m}\right)[[t]]$ is positive in $z_{j}$ (or $z_{j}$-positive) if, in every term of its $z$-expansion, $z_{j}$ appears with a positive exponent. We define similarly the notion of $z_{j}$-negative, $z_{j}$-non-positive, $z_{j}$-non-negative series. We have just observed that, for $m=5$ and $\sigma=13425$, the series $\sigma(M(x) \bar{F}(x ; t)) / M(x)$ is non-negative in $z_{2}$ but negative in $z_{4}$. This is generalized by the following lemma.

Lemma 14 Take $\sigma \in \mathfrak{S}_{m} \backslash\{\mathrm{id}\}$. Let $\sigma(j)$ be the largest non-fixed left-to-right maximum of $\sigma$. That is,

for $k<j, \sigma(k)<\sigma(j)$, and for every $k$ such that $\sigma(k)>\sigma(j)$, one has $\sigma(k)=k$.

Let $\sigma(i)$ be any value that is not a left-to-right maximum and satisfies $\sigma(i) \leq i$. For $e_{1} \geq 0, \ldots, e_{m} \geq 0$, consider the fraction

$$
\frac{1}{M(x)} \sigma\left(\frac{M(x)}{x_{1}^{e_{1}}\left(x_{1}+x_{2}\right)^{e_{2}} \cdots\left(x_{1}+\cdots+x_{m}\right)^{e_{m}}}\right) .
$$

Then this fraction is non-negative in $z_{\sigma(i)}$ but negative in $z_{\sigma(j)}$. Since $\sigma(i)<\sigma(j)$, applying $\Lambda$ to this fraction gives 0 .

Returning to the example $\sigma=13425$ studied above, we observe that the lemma applies with $\sigma(i)=2$ and $\sigma(j)=4$.

This lemma implies the second part of (31): indeed, the contribution of any $\tau \in$ $\mathfrak{S}^{(m)}$ in $\sigma(M(x) \bar{F}(x ; t)) / M(x)$ is of the form (33). Hence proving the lemma will conclude the proof of Proposition 13.

Proof of Lemma 14 We establish this lemma via a sequence of three elementary properties.

Property 1 Let $i_{1}<i_{2}<\cdots<i_{k}$, and $e \in \mathbb{Z}$. The fraction

$$
\frac{1}{\left( \pm z_{i_{1}} \pm \cdots \pm z_{i_{k}}\right)^{e}}
$$

is non-negative in $z_{i_{1}}, \ldots, z_{i_{k-1}}$. If $e \geq 0$, it is non-positive in $z_{i_{k}}$, and even negative in $z_{i_{k}}$ if $e>0$.

Proof The result is obvious if $e \leq 0$, as the fraction is a polynomial in this case. If $e>0$, we prove it by induction on $k$. It clearly holds for $k=1$. If $k>1$, we write

$$
\begin{aligned}
\frac{1}{\left( \pm z_{i_{1}} \pm \cdots \pm z_{i_{k}}\right)^{e}} & =\frac{1}{\left( \pm z_{i_{2}} \pm \cdots \pm z_{i_{k}}\right)^{e}\left(1 \pm \frac{z_{i_{1}}}{z_{i_{2}} \pm \cdots \pm z_{i_{k}}}\right)^{e}} \\
& =\sum_{n \geq 0}\left(\begin{array}{c}
e-1+n \\
n
\end{array}\right) \frac{\left( \pm z_{i_{1}}\right)^{n}}{\left( \pm z_{i_{2}} \pm \cdots \pm z_{i_{k}}\right)^{e+n}}
\end{aligned}
$$

and we conclude by induction on $k$. 
Property 2 Let $\sigma, j, e_{1}, \ldots, e_{m}$ be as in Lemma 14. The fraction

$$
\sigma\left(\frac{1}{x_{1}^{e_{1}}\left(x_{1}+x_{2}\right)^{e_{2}} \cdots\left(x_{1}+\cdots+x_{m}\right)^{e_{m}}}\right)
$$

is non-negative in all $z_{\sigma(k)}$ such that $\sigma(k)$ is not a left-to-right maximum, and nonpositive in $z_{\sigma(j)}$.

Proof It suffices to prove that the result holds for each term

$$
\sigma\left(\frac{1}{\left(x_{1}+\cdots+x_{\ell}\right)^{e_{\ell}}}\right)=\frac{1}{\left(z_{\sigma(1)}-z_{\sigma(1)-1}+\cdots+z_{\sigma(\ell)}-z_{\sigma(\ell)-1}\right)^{e_{\ell}}},
$$

for $\ell \in\{1, \ldots, m\}$ (with $z_{0}=0$ ).

By Property 1 , this term is non-negative in all variables, except possibly in $z_{\max }(\sigma(1), \ldots, \sigma(\ell))$. Since $\max (\sigma(1), \ldots, \sigma(\ell))$ is always a left-to-right maximum, this proves the first part of the property.

Consider now the variable $z_{\sigma(j)}$.

- If $\ell<j$, then $\max (\sigma(1), \ldots, \sigma(\ell))<\sigma(j)$, so that the term (34) is independent of $z_{\sigma(j)}$, and thus non-positive in this variable.

- If $j \leq \ell \leq \sigma(j)$, then $\max (\sigma(1), \ldots, \sigma(\ell))=\sigma(j)$. Then (34) is non-positive in $z_{\sigma(j)}$ by Property 1 .

- Finally, if $\ell>\sigma(j)$, then $\{\sigma(1), \ldots, \sigma(\ell)\}=\{1, \ldots, \ell\}$, so that the term (34) simply reads $1 / z_{\ell}^{e_{\ell}}$. This is independent of $z_{\sigma(j)}$, and thus non-positive in this variable.

Property 3 Let $\sigma$ and $j$ be as in Lemma 14. The fraction

$$
\frac{\sigma(M(x))}{M(x)}
$$

is non-negative in all $z_{\sigma(k)}$ such that $\sigma(k) \leq k$, and negative in $z_{\sigma(j)}$.

Proof We have

$$
\frac{\sigma(M(x))}{M(x)}=\prod_{\ell=1}^{m}\left(z_{\sigma(\ell)}-z_{\sigma(\ell)-1}\right)^{\ell-\sigma(\ell)} .
$$

Assume $\sigma(k) \leq k$. The two terms of the above product that involve $z_{\sigma(k)}$ are $\left(z_{\sigma(k)}-\right.$ $\left.z_{\sigma(k)-1}\right)^{k-\sigma(k)}$ and $\left(z_{\sigma(k)+1}-z_{\sigma(k)}\right)^{e}$, with $e=\sigma^{-1}(\sigma(k)+1)-\sigma(k)-1$. The former term is non-negative in $z_{\sigma(k)}$ because $\sigma(k) \leq k$. The latter term is non-negative in $z_{\sigma(k)}$ by Property 1 . This proves the first part of the property.

The two terms that involve $z_{\sigma(j)}$ are $\left(z_{\sigma(j)}-z_{\sigma(j)-1}\right)^{j-\sigma(j)}$ and $\left(z_{\sigma(j)+1}-z_{\sigma(j)}\right)^{e}$, with $e=\sigma^{-1}(\sigma(j)+1)-\sigma(j)-1$. Since $\sigma(j)>j$, the former term is negative in $z_{\sigma(j)}$ by Property 1 . By construction of $j$, the exponent $e$ is 0 , so that the latter term is simply 1 .

Lemma 14 now follows by combining Properties 2 and 3 . 


\subsection{Determinantal expression of the series}

Let us write $e^{t \bar{x}}=\sum_{b \geq 0}(t \bar{x})^{b} / b$ !. The second formula in Proposition 13 reads:

$$
\sum_{\tau \in \mathfrak{S}^{(m)}} \frac{t^{|\tau|}}{|\tau| !}=\sum_{b_{1}, \ldots, b_{m} \geq 0} \frac{t^{b_{1}+\cdots+b_{m}}}{b_{1} ! \cdots b_{m} !} \sum_{\sigma \in \mathfrak{S}_{m}} \varepsilon(\sigma) \Lambda\left(\frac{\sigma(M(x))}{M(x) \sigma\left(x^{b}\right)}\right)
$$

where $M(x)=x_{2} x_{3}^{2} \cdots x_{m}^{m-1}, b=\left(b_{1}, \ldots, b_{m}\right)$, and $x^{b}=x_{1}^{b_{1}} \cdots x_{m}^{b_{m}}$. We will give a closed form expression of $\Lambda\left(\frac{\sigma(M(x))}{M(x) \sigma\left(x^{b}\right)}\right)$ (Lemma 17), which in turn will give a closed form expression of the sum over $\sigma$ occurring in (35).

Proposition 15 For $b=\left(b_{1}, \ldots, b_{m}\right) \in \mathbb{N}^{m}$,

$$
\sum_{\sigma \in \mathfrak{S}_{m}} \varepsilon(\sigma) \Lambda\left(\frac{\sigma(M(x))}{M(x) \sigma\left(x^{b}\right)}\right)=\frac{\left(b_{1}+\cdots+b_{m}\right) !}{\prod_{i=1}^{m}\left(b_{i}-i+m\right) !} \prod_{1 \leq i<j \leq m}\left(b_{i}-i-b_{j}+j\right) .
$$

Let us delay for the moment the proof of this proposition, and derive from it Gessel's determinantal formula (Theorem 1).

Proof of Theorem 1 The exponential generating function of permutations of $\mathfrak{S}^{(m)}$ now reads

$$
\sum_{\tau \in \mathfrak{S}^{(m)}} \frac{t^{|\tau|}}{|\tau| !}=\sum_{b_{1}, \ldots, b_{m} \geq 0} t^{b_{1}+\cdots+b_{m}} \frac{\left(b_{1}+\cdots+b_{m}\right) !}{\prod_{i=1}^{m} b_{i} !\left(b_{i}-i+m\right) !} \prod_{1 \leq i<j \leq m}\left(b_{i}-i-b_{j}+j\right) .
$$

Replacing $t$ by $t^{2}$, and taking the Bessel generating function gives

$$
\sum_{\tau \in \mathfrak{S}^{(m)}} \frac{t^{2|\tau|}}{|\tau| !^{2}}=\sum_{b_{1}, \ldots, b_{m} \geq 0} \frac{t^{2\left(b_{1}+\cdots+b_{m}\right)}}{\prod_{i=1}^{m} b_{i} !\left(b_{i}-i+m\right) !} \prod_{1 \leq i<j \leq m}\left(b_{i}-i-b_{j}+j\right) .
$$

But this is exactly Gessel's determinant. Indeed:

$$
\begin{aligned}
& \operatorname{det}\left(I_{j-i}\right)_{1 \leq i, j \leq m} \\
& =\sum_{\sigma \in \mathfrak{S}_{m}} \varepsilon(\sigma) \prod_{i=1}^{m} I_{\sigma(i)-i} \\
& =\sum_{\sigma \in \mathfrak{S}_{m}} \varepsilon(\sigma) \prod_{i=1}^{m} \sum_{b_{i} \geq 0} \frac{t^{2 b_{i}-i+\sigma(i)}}{b_{i} !\left(b_{i}-i+\sigma(i)\right) !} \\
& =\sum_{b_{1}, \ldots, b_{m} \geq 0} \frac{t^{2\left(b_{1}+\cdots+b_{m}\right)}}{\prod_{i=1}^{m} b_{i} !\left(b_{i}-i+m\right) !} \operatorname{det}\left(\left(b_{i}-i+j+1\right) \cdots\left(b_{i}-i+m\right)\right),
\end{aligned}
$$

and this coincides with (36), because the above determinant is the Vandermonde determinant in the variables $u_{i}:=b_{i}-i$ (since $\left(b_{i}-i+j+1\right) \cdots\left(b_{i}-i+m\right)$ is a polynomial in $u_{i}$ of dominant term $u_{i}^{m-j}$ ). 
There remains to prove Proposition 15. The proof relies on two lemmas. The first one is a simple identity based on a partial fraction expansion. The second gives a closed form expression of $\Lambda\left(\frac{\sigma(M(x))}{M(x) \sigma\left(x^{b}\right)}\right)$, for $b \in \mathbb{N}^{m}$.

Lemma 16 Let $x_{1}, \ldots, x_{k}, u_{1}, \ldots, u_{k}$ be indeterminates, and let the symmetric group $\mathfrak{S}_{k}$ act on the $x_{i}$ 's by permuting them (that is, $\tau\left(x_{i}\right)=x_{\tau(i)}$ for $\tau \in \mathfrak{S}_{k}$ ). Then

$$
\sum_{\tau \in \mathfrak{S}_{k}} \varepsilon(\tau) \tau\left(\prod_{i=1}^{k-1} \frac{\left(x_{i}+u_{i}\right) \cdots\left(x_{i}+u_{k}\right)}{x_{i}+u_{i}+\cdots+x_{k}+u_{k}}\right)=\prod_{1 \leq i<j \leq k}\left(x_{i}-x_{j}\right) .
$$

Proof Let us denote $u=\left(u_{1}, \ldots, u_{k}\right)$ and

$$
T(x, u)=\prod_{i=1}^{k-1} \frac{\left(x_{i}+u_{i}\right) \cdots\left(x_{i}+u_{k}\right)}{x_{i}+u_{i}+\cdots+x_{k}+u_{k}}
$$

This is a rational function of $u_{k}$, in which the numerator and denominator have degree $k-1$. By a partial fraction expansion,

$$
T(x, u)=C(x, u)+\sum_{\ell=1}^{k-1} \frac{\alpha_{\ell}(x, u)}{x_{\ell}+u_{\ell}+\cdots+x_{k}+u_{k}},
$$

where $C$ and the $\alpha_{\ell}$ 's are independent of $u_{k}$. By letting $u_{k}$ tend to infinity, one obtains

$$
C(x, u)=\prod_{1 \leq i \leq j<k}\left(x_{i}+u_{j}\right) \text {. }
$$

The value of $\alpha_{\ell}$ is obtained by taking the residue of $T(x, u)$ at $u_{k}=-\left(x_{\ell}+u_{\ell}+\right.$ $\left.\cdots+x_{k}\right)$. This gives, for $\ell \leq k-1$ :

$$
\alpha_{\ell}(x, u)=\frac{\prod_{1 \leq i \leq j<k}\left(x_{i}+u_{j}\right) \prod_{1 \leq i<k}\left(x_{i}-\left(x_{\ell}+u_{\ell}+\cdots+x_{k}\right)\right)}{\prod_{i \neq \ell, i<k}\left(x_{i}+u_{i}+\cdots+x_{k-1}+u_{k-1}+x_{k}-\left(x_{\ell}+u_{\ell}+\cdots+x_{k}\right)\right)} .
$$

Return now to (37). It is easy to check that $\alpha_{\ell}(x, u) /\left(x_{\ell}+u_{\ell}+\cdots+x_{k}+u_{k}\right)$ is left unchanged by the exchange of $x_{\ell}$ and $x_{\ell+1}$. Consequently, the sum of the lemma reads

$$
\begin{aligned}
\sum_{\tau \in \mathfrak{S}_{k}} \varepsilon(\tau) \tau(T(x, u)) & =\sum_{\tau \in \mathfrak{S}_{k}} \varepsilon(\tau) \tau(C(x, u))=\operatorname{det}\left(\prod_{h=i}^{k-1}\left(x_{j}+u_{h}\right)\right)_{1 \leq i, j \leq k} \\
& =\prod_{1 \leq i<j \leq k}\left(x_{i}-x_{j}\right),
\end{aligned}
$$

because $\prod_{h=i}^{k-1}\left(x_{j}+u_{h}\right)$ is a polynomial in $x_{j}$ of leading term $x_{j}^{k-i}$; the sum over $\tau$ thus reduces to a Vandermonde determinant. 
Lemma 17 Let $b=\left(b_{1}, \ldots, b_{m}\right) \in \mathbb{N}^{m}$ and $\sigma \in \mathfrak{S}_{m}$. Let

$$
\frac{1}{x^{e}}=\frac{\sigma(M(x))}{M(x) \sigma\left(x^{b}\right)},
$$

where as before $M(x)=x_{2} \cdots x_{m}^{m-1}$. That is, $e=\left(e_{1}, \ldots, e_{m}\right)$ where $e_{i}=b_{\tau(i)}-$ $\tau(i)+i$ and $\tau=\sigma^{-1}$. Let $k=\max \left\{i: b_{i}>0\right\}($ if $b=(0, \ldots, 0)$, we take $k=0)$. Then

$$
\Lambda\left(\frac{1}{x^{e}}\right)= \begin{cases}\prod_{i=1}^{k}\left(\begin{array}{c}
e_{i}+\cdots+e_{k}-1 \\
e_{i}-1
\end{array}\right), & \text { if } \sigma(j)=j \text { for all } j>k \\
0, & \text { otherwise. }\end{cases}
$$

Remark If $\sigma(j)=j$ for all $j>k$, and $i \leq k$, then $e_{i}+\cdots+e_{k} \geq 1$. Indeed, if $e_{i}+\cdots+e_{k}=b_{\tau(i)}+\cdots+b_{\tau(k)}+(i+\cdots+k)-(\tau(i)+\cdots+\tau(k))$ were nonpositive, this would mean that $\{\tau(i), \ldots, \tau(k)\}=\{i, \ldots, k\}$ and $b_{i}=\cdots=b_{k}=0$, which contradicts the definition of $k$. However, $e_{i}$ may be non-positive, and in this case the above expression vanishes. However, $e_{i}+k-i \geq 0$. When we apply this lemma to prove Proposition 15, we will write the above product of binomial coefficients in the following equivalent form:

$$
\frac{\left(e_{1}+\cdots+e_{k}\right) !}{\prod_{1 \leq i \leq k}\left(e_{i}+k-i\right) !} \prod_{i=1}^{k-1} \frac{e_{i}\left(e_{i}+1\right) \cdots\left(e_{i}+k-i\right)}{e_{i}+\cdots+e_{k}} .
$$

Proof For an iterated Laurent series in $z_{1}, \ldots, z_{k}$, of the form $R(z)=$ $\sum_{n \in \mathbb{Z}^{k}} c\left(n_{1}, \ldots, n_{k}\right) z_{1}^{n_{1}} \cdots z_{k}^{n_{k}}$, we define the negative part of $R(z)$ by

$$
\left[z^{<}\right] R(z)=\left[z_{1}^{<} \cdots z_{k}^{<}\right] R(z):=\sum_{n_{1}<0, \ldots, n_{k}<0} c\left(n_{1}, \ldots, n_{k}\right) .
$$

Let us first prove that if $f=\left(f_{1}, \ldots, f_{k}\right) \in \mathbb{Z}^{k}$,

$$
\left[z^{<}\right]\left(\frac{1}{x^{f}}\right)=\prod_{i=1}^{k}\left(\begin{array}{c}
f_{i}+\cdots+f_{k}-1 \\
f_{i}-1
\end{array}\right) \text {. }
$$

We adopt the standard convention that $\left(\begin{array}{l}a \\ b\end{array}\right)=0$ unless $0 \leq b \leq a$. Given that $x_{i}=$ $z_{i}-z_{i-1}$, we have

$$
\frac{1}{x^{f}}=\frac{1}{z_{1}^{f_{1}} \cdots z_{k}^{f_{k}}\left(1-\frac{z_{1}}{z_{2}}\right)^{f_{2}} \cdots\left(1-\frac{z_{k-1}}{z_{k}}\right)^{f_{k}}} .
$$

If $f_{i} \leq 0$ for some $i$, the $z$-expansion of $1 / x^{f}$ only involves non-negative powers of $z_{i}$, so that the negative part of $1 / x^{f}$ is zero. The right-hand side of (40) is zero as well, and thus (40) holds. Assume now $f_{i}>0$ for all $i$. The proof works by induction on $k$. If $k=1$ and $f_{1}>0$,

$$
\left[z^{<}\right]\left(\frac{1}{z_{1}^{f_{1}}}\right)=1=\left(\begin{array}{c}
f_{1}-1 \\
f_{1}-1
\end{array}\right) .
$$


For $k \geq 2$,

$$
\begin{aligned}
{\left[z^{<}\right]\left(\frac{1}{x^{f}}\right)=} & {\left[z^{<}\right] \frac{1}{z_{1}^{f_{1}} z_{2}^{f_{2}}\left(1-\frac{z_{1}}{z_{2}}\right)^{f_{2}}\left(z_{3}-z_{2}\right)^{f_{3}} \cdots\left(z_{k}-z_{k-1}\right)^{f_{k}}} } \\
= & {\left[z^{<}\right] \sum_{n \geq 0}\left(\begin{array}{c}
n+f_{2}-1 \\
f_{2}-1
\end{array}\right) \frac{z_{1}^{n-f_{1}}}{z_{2}^{n+f_{2}}\left(z_{3}-z_{2}\right)^{f_{3}} \cdots\left(z_{k}-z_{k-1}\right)^{f_{k}}} } \\
= & \sum_{n=0}^{f_{1}-1}\left(\begin{array}{c}
n+f_{2}-1 \\
f_{2}-1
\end{array}\right)\left[z_{2}^{<} \cdots z_{k}^{<}\right] \frac{1}{z_{2}^{n+f_{2}}\left(z_{3}-z_{2}\right)^{f_{3}} \cdots\left(z_{k}-z_{k-1}\right)^{f_{k}}} \\
= & \sum_{n=0}^{f_{1}-1}\left(\begin{array}{c}
n+f_{2}-1 \\
f_{2}-1
\end{array}\right)\left(\begin{array}{c}
n+f_{2}+\cdots+f_{k}-1 \\
n+f_{2}-1
\end{array}\right) \prod_{i=3}^{k}\left(\begin{array}{c}
f_{i}+\cdots+f_{k}-1 \\
f_{i}-1
\end{array}\right)
\end{aligned}
$$

by the induction hypothesis. Now

$$
\begin{aligned}
& \sum_{n=0}^{f_{1}-1}\left(\begin{array}{c}
n+f_{2}-1 \\
f_{2}-1
\end{array}\right)\left(\begin{array}{c}
n+f_{2}+\cdots+f_{k}-1 \\
n+f_{2}-1
\end{array}\right) \\
& =\sum_{n=0}^{f_{1}-1}\left(\begin{array}{c}
n+f_{2}+\cdots+f_{k}-1 \\
n
\end{array}\right)\left(\begin{array}{c}
f_{2}+\cdots+f_{k}-1 \\
f_{2}-1
\end{array}\right) \\
& =\left(\begin{array}{c}
f_{1}+f_{2}+\cdots+f_{k}-1 \\
f_{1}-1
\end{array}\right)\left(\begin{array}{c}
f_{2}+\cdots+f_{k}-1 \\
f_{2}-1
\end{array}\right) .
\end{aligned}
$$

The last equality results from the classical binomial identity

$$
\sum_{n=0}^{a}\left(\begin{array}{c}
n+b \\
n
\end{array}\right)=\left(\begin{array}{c}
a+b+1 \\
a
\end{array}\right)
$$

This gives (40).

Let us now prove (38). Assume that $\sigma(j)=j$ for all $j>k$. This implies that $e_{k+1}=\cdots=e_{m}=0$. As argued just after the statement of the lemma, $e_{k}>0$. But then $1 / x^{e}$ is negative in $z_{k}$, and involves none of the variables $z_{k+1}, \ldots, z_{m}$. Thus, by definition of $\Lambda$,

$$
\Lambda\left(\frac{1}{x^{e}}\right)=\left[z_{1}^{<} \cdots z_{k}^{<}\right] \frac{1}{x_{1}^{e_{1}} \cdots x_{k}^{e_{k}}}=\prod_{i=1}^{k}\left(\begin{array}{c}
e_{i}+\cdots+e_{k}-1 \\
e_{i}-1
\end{array}\right)
$$

(by (40)), and this gives the first part of (38).

Assume now that there exists $j>k$ such that $\sigma(j) \neq j$. Then there also exists $j>k$ such that $\sigma(j)<j$. Let us choose such a $j$. Then there also exists $\ell>\sigma(j)$ such that $\tau(\ell)<\ell$. We have

$$
e_{\sigma(j)}=b_{j}-j+\sigma(j)=-j+\sigma(j)<0,
$$




$$
e_{\ell}=b_{\tau(\ell)}-\tau(\ell)+\ell>0,
$$

with $\ell>\sigma(j)$. Let $\ell^{\prime}=\max \left\{p>\sigma(j): e_{p}>0\right\}$ (this set is non-empty as it contains $\ell)$. Then $e_{\ell^{\prime}+1}=\cdots=e_{m}=0$, and $1 / x^{e}$ is non-negative in $z_{\sigma(j)}$ but negative in $z_{\ell^{\prime}}$. By definition of $\Lambda$, this implies that $\Lambda\left(1 / x^{e}\right)=0$.

Proof of Proposition 15 Let us denote by $\operatorname{SUM}(b)$ the sum we want to evaluate. Let $k=\max \left\{i: b_{i}>0\right\}$. By Lemma 17, the sum can be reduced to permutations $\sigma$ that fix all points larger than $k$, and then we use the closed form expression (39) of $\Lambda\left(\frac{\sigma(M(x))}{M(x) \sigma\left(x^{b}\right)}\right)$. This gives:

$$
\begin{aligned}
\operatorname{SUM}(b)= & \sum_{\sigma \in \mathfrak{S}_{k}} \varepsilon(\sigma) \Lambda\left(\frac{\sigma(M(x))}{M(x) \sigma\left(x^{b}\right)}\right) \\
= & \sum_{\tau \in \mathfrak{S}_{k}} \varepsilon(\tau) \frac{\left(b_{1}+\cdots+b_{k}\right) !}{\prod_{i=1}^{k}\left(b_{\tau(i)}-\tau(i)+k\right) !} \\
& \times \prod_{i=1}^{k-1} \frac{\left(b_{\tau(i)}-\tau(i)+i\right) \cdots\left(b_{\tau(i)}-\tau(i)+k\right)}{b_{\tau(i)}-\tau(i)+i+\cdots+b_{\tau(k)}-\tau(k)+k} \\
= & \frac{\left(b_{1}+\cdots+b_{k}\right) !}{\prod_{i=1}^{k}\left(b_{i}-i+k\right) !} \prod_{1 \leq i<j \leq k}\left(b_{i}-i-b_{j}+j\right) .
\end{aligned}
$$

The last equality is the case $x_{i}=b_{i}-i, u_{i}=i$ of Lemma 16 . It is easy to check that, given that $b_{k+1}=\cdots=b_{m}=0$, the above expression coincides with

$$
\frac{\left(b_{1}+\cdots+b_{m}\right) !}{\prod_{i=1}^{m}\left(b_{i}-i+m\right) !} \prod_{1 \leq i<j \leq m}\left(b_{i}-i-b_{j}+j\right),
$$

as stated in Proposition 15.

\section{Final comments}

Clearly, our proof of Theorem 1, dealing with permutations of $\mathfrak{S}^{(m)}$, is more complicated than our proof of Theorem 2, dealing with involutions of $\mathfrak{I}^{(m)}$. We still wonder if there exists another change of variables, another coefficient extraction or another way to perform this extraction effectively that would simplify Sects. 5.3 and 5.4.

Our approach is robust enough to be adapted to other enumeration problems. Consider for instance the set $\tilde{\mathfrak{S}}^{(m)}$ of permutations $\pi$ of $\mathfrak{S}^{(m)}$ in which the values $1,2, \ldots, m$ occur in this order. That is, $\pi^{-1}(1)<\cdots<\pi^{-1}(m)$. Garsia and Goupil found recently a simple formula for the number of such permutations of (small) length $n$ : if $n \leq 2 m$, this number is [15, Corollary 6.2]

$$
\sharp \tilde{S}_{n}^{(m)}=\sum_{r=0}^{n-m}(-1)^{r}\left(\begin{array}{c}
n-m \\
r
\end{array}\right) \frac{n !}{(m+r) !} .
$$


This was then reproved by Panova [32]. See also [1].

In terms of the generating tree described in Sect. 2.1, this means that one is only counting the nodes of the subtree rooted at the permutation $12 \cdots m$. The only change in the functional equation of Proposition 5 is thus the initial condition: instead of 1 (which accounts for the empty permutation), it is now $v_{1} \cdots v_{m} t^{m}$. Sections 5.1 to 5.3 translate verbatim, and we reach the following counterpart of Proposition 13.

Proposition 18 The ordinary generating function of permutations that avoid $12 \cdots m(m+1)$ and in which the values $1, \ldots, m$ occur in this order is obtained by applying the operator $\Lambda$ of Definition 12 to a rational function:

$$
\begin{aligned}
\sum_{\tau \in \tilde{\mathfrak{S}}^{(m)}} t^{|\tau|}= & \Lambda\left(\frac{t^{m}}{x_{2}^{1} \cdots x_{m}^{m-1}\left(1-t\left(\bar{x}_{1}+\cdots+\bar{x}_{m}\right)\right)}\right. \\
& \left.\times \sum_{\sigma \in \mathfrak{S}_{m}} \epsilon(\sigma) \sigma\left(\frac{x_{2}^{1} \cdots x_{m}^{m-1}}{\prod_{i=1}^{m}\left(x_{1}+\cdots+x_{i}\right)}\right)\right),
\end{aligned}
$$

with $x_{j}=z_{j}-z_{j-1}$ and $z_{0}=0$.

Equivalently, the exponential generating function of these permutations is

$$
\sum_{\tau \in \tilde{\mathfrak{S}}^{(m)}} \frac{t^{|\tau|}}{|\tau| !}=\Lambda\left(\frac{t^{m} e^{t\left(\bar{x}_{1}+\cdots+\bar{x}_{m}\right)}}{x_{2}^{1} \cdots x_{m}^{m-1}} \sum_{\sigma \in \mathfrak{S}_{m}} \epsilon(\sigma) \sigma\left(\frac{x_{2}^{1} \cdots x_{m}^{m-1}}{\prod_{i=1}^{m}\left(x_{1}+\cdots+x_{i}\right)}\right)\right) .
$$

We have not further pursued in this direction, but one could try to obtain a more explicit formula giving the whole generating function (which would imply (41) when $n \leq 2 m)$.

As discussed at the beginning of Sect. 2.2, the generating tree for $12 \cdots m(m+1)$ avoiding involutions can be described using $m$ catalytic variables. Since these involutions are equinumerous with $(m+1) m \cdots 21$-avoiding involutions, it is natural to ask whether one could derive Theorem 2 from this tree and the corresponding functional equation. This could allow us to address the enumeration of $12 \cdots m(m+1)$-avoiding fixed point free involutions, for which determinantal formulas exist (obtained by applying Gessel's $\theta$ operator [16] to identities (5.41) and (5.42) of [3], which are equivalent to Theorem 2.3(3) in [31]; see also Stanley's survey [39, Thm. 8]). The recursive construction we have used for involutions does not allow us to address this problem.

Acknowledgements The author thanks Aaron Jaggard for communicating an early version of his paper with Joseph Marincel [21].

\section{References}

1. Amdeberhan, T.: A note on a question due to A. Garsia. In: Amdeberhan, T., Medina, L.A., Moll, V.H. (eds.) Gems in Experimental Mathematics. Contemp. Math., vol. 517. Amer. Math. Soc., Providence (2010). ArXiv:0906.0566 [math.CO]

2. Backelin, J., West, J., Xin, G.: Wilf-equivalence for singleton classes. Adv. Appl. Math. 38(2), 133148 (2007) 
3. Baik, J., Rains, E.M.: Algebraic aspects of increasing subsequences. Duke Math. J. 109(1), 1-65 (2001)

4. Baik, J., Deift, P., Johansson, K.: On the distribution of the length of the longest increasing subsequence of random permutations. J. Am. Math. Soc. 12(4), 1119-1178 (1999)

5. Banderier, C., Bousquet-Mélou, M., Denise, A., Flajolet, P., Gardy, D., Gouyou-Beauchamps, D.: Generating functions for generating trees. Discrete Math. 246(1-3), 29-55 (2002)

6. Bender, E.A., Knuth, D.E.: Enumeration of plane partitions. J. Comb. Theory Ser. A 13, 40-54 (1972)

7. Bloom, J., Saracino, D.: On bijections for pattern-avoiding permutations. J. Comb. Theory Ser. A 116(8), 1271-1284 (2009)

8. Bousquet-Mélou, M.: Four classes of pattern-avoiding permutations under one roof: Generating trees with two labels. Electron. J. Comb. 9(2), Research Paper 19, 31 pp. (electronic) (2003)

9. Bousquet-Mélou, M.: Walks in the quarter plane: Kreweras' algebraic model. Ann. Appl. Probab. 15(2), 1451-1491 (2005)

10. Bousquet-Mélou, M., Mishna, M.: Walks with small steps in the quarter plane. Contemp. Math. 520, $1-40(2010)$

11. Bousquet-Mélou, M., Petkovšek, M.: Linear recurrences with constant coefficients: the multivariate case. Discrete Math. 225(1-3), 51-75 (2000)

12. Bousquet-Mélou, M., Steingrímsson, E.: Decreasing subsequences in permutations and Wilf equivalence for involutions. J. Algebr. Comb. 22(4), 383-409 (2005)

13. Dukes, W.M.B., Jelínek, V., Mansour, T., Reifegerste, A.: New equivalences for pattern avoidance for involutions. Proc. Am. Math. Soc. 137, 457-465 (2009)

14. Elizalde, S., Pak, I.: Bijections for refined restricted permutations. J. Comb. Theory Ser. A 105(2), 207-219 (2004)

15. Garsia, A.M., Goupil, A.: Character polynomials, their $q$-analogs and the Kronecker product. Electron. J. Comb. 16(2), Research Paper 19, 40 pp. (electronic) (2009)

16. Gessel, I.M.: Symmetric functions and P-recursiveness. J. Comb. Theory Ser. A 53(2), 257-285 (1990)

17. Gessel, I.M., Zeilberger, D.: Random walk in a Weyl chamber. Proc. Am. Math. Soc. 115(1), 27-31 (1992)

18. Gessel, I.M., Weinstein, J., Wilf, H.S.: Lattice walks in $\mathbf{Z}^{d}$ and permutations with no long ascending subsequences. Electron. J. Comb. 5(1), Research Paper 2, 11 pp. (electronic) (1998)

19. Goulden, I.P.: A linear operator for symmetric functions and tableaux in a strip with given trace. Discrete Math. 99(1-3), 69-77 (1992)

20. Guibert, O.: Combinatoire des permutations à motifs exclus, en liaison avec mots, cartes planaires et tableaux de Young. PhD thesis, LaBRI, Université Bordeaux 1 (1995)

21. Jaggard, A.D., Marincel, J.J.: Generating tree isomorphisms for pattern-avoiding involutions. Ann. Comb. (to appear). Available at http://dimacs.rutgers.edu/ adj/Research/jm_invols_071201.pdf

22. Knuth, D.E.: The Art of Computer Programming. Sorting and Searching, vol. 3. Addison-Wesley, Reading (1968)

23. Krattenthaler, C.: Non-crossing two-rowed arrays and summations for Schur functions. In: Barlotti, A., Delest, M., Pinzani, R. (eds.) Proc. of the 5th Conference on Formal Power Series and Algebraic Combinatorics, pp. 301-314. Florence, Italy (1993)

24. Krattenthaler, C.: Advanced determinant calculus. Sém. Lothar. Comb. 42 (The Andrews Festschrift), Article B42q, 67 pp. (1999)

25. Krattenthaler, C.: Permutations with restricted patterns and Dyck paths. Adv. Appl. Math. 27(2-3), 510-530 (2001)

26. Krattenthaler, C.: Growth diagrams, and increasing and decreasing chains in fillings of Ferrers shapes. Adv. Appl. Math. 37, 404-431 (2006)

27. Lipshitz, L.: The diagonal of a $D$-finite power series is $D$-finite. J. Algebra 113(2), 373-378 (1988)

28. Lipshitz, L.: D-finite power series. J. Algebra 122, 353-373 (1989)

29. MacMahon, P.A.: Combinatory Analysis. Vol. I, II (bound in one volume). Dover, Mineola (2004). Reprint of An Introduction to Combinatory Analysis (1920) and Combinatory Analysis. Vol. I, II $(1915,1916)$

30. Novak, J.: Vicious walkers and random contraction matrices. Int. Math. Res. Not. IMRN (17), 33103327 (2009)

31. Okada, S.: Applications of minor summation formulas to rectangular-shaped representations of classical groups. J. Algebra 205(2), 337-367 (1998) 
32. Panova, G.: Bijective enumeration of permutations starting with a longest increasing subsequence. In: FPSAC 10, MTCS Proceedings, San Francisco, USA, pp. 973-983 (2010). arXiv:0905.2013 [math.CO]

33. Prodinger, H.: The kernel method: a collection of examples. Sém. Lothar. Comb. 50, Art. B50f, 19 pp. (electronic) (2003/04)

34. Robertson, A.: Restricted permutations from Catalan to Fine and back. Sém. Lothar. Comb. 50, Art. B50g, 13 pp. (electronic) (2004)

35. Robertson, A., Saracino, D., Zeilberger, D.: Refined restricted permutations. Ann. Comb. 6(3-4), 427-444 (2002)

36. Rotem, D.: On a correspondence between binary trees and a certain type of permutation. Inf. Process. Lett. 4, 58-61 (1975)

37. Schensted, C.: Longest increasing and decreasing subsequences. Can. J. Math. 13, 179-191 (1961)

38. Stanley, R.P.: Enumerative Combinatorics, vol. 2. Cambridge Studies in Advanced Mathematics, vol. 62. Cambridge University Press, Cambridge (1999)

39. Stanley, R.P.: Increasing and decreasing subsequences and their variants. In: International Congress of Mathematicians, vol. I, pp. 545-579. Eur. Math. Soc., Zürich (2007)

40. West, J.: Generating trees and the Catalan and Schröder numbers. Discrete Math. 146(1-3), 247-262 (1995)

41. West, J.: Generating trees and forbidden subsequences. Discrete Math. 157(1-3), 363-374 (1996)

42. Xin, G.: Determinant formulas relating to tableaux of bounded height. Adv. Appl. Math. 45(2), 197211 (2010). arXiv:0704.3381 